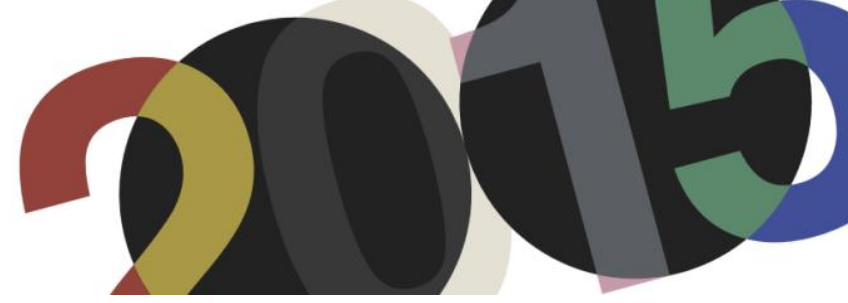

DOI: http://dx.doi.org/10.4995/LC2015.2015.1025

\title{
Machines à exposer
}

\author{
C. Dumont d'Ayot
}

Institut für Denkmalpflege und Bauforschung, ETH Zurich

Résumé: Ateliers d'artistes, appartements et villas de collectionneurs, pavillons, scénographies et musées : l'exposition est un fil rouge de l'œuvre de Le Corbusier. Le rapport que l'homme entretient à l'œuvre d'art et les modalités de ce rapport sont des éléments fondateurs de son architecture et occupent une position primordiale dans sa vision de la ville. De la ziggourat du Musée mondial en 1929, jusqu'aux projets des années 1960 comme le Centre d'Art international à Erlenbach ou le Musée du XXe siècle pour Nanterre, les musées sont des pièces incontournables des ses grands plans d'urbanisme. Les projets de musées et de pavillons d'exposition entre 1929 et 1965 et les concepts des différentes expositions qu'il organise évoluent en parallèle de sa manière d'envisager le rapport à l'œuvre, que ce soit celui de l'artiste, du spectateur initié ou du novice. Les esquisses préparatoires des différents projets de musées et de pavillons retracent cette évolution. La critique du projet du Mundaneum par Karel Teige assume un rôle clé dans la transformation décisive du concept du musée qui a lieu entre le Musée Mondial en 1929 et le projet de Le Corbusier pour le Musée à croissance illimitée en 1930. C'est un changement séminal qui est décisif pour les projets futurs. L'architecture et la relation à l'œuvre d'art ne sont plus déterminées par le recours à une forme, mais par un mécanisme fonctionnel et organique: la croissance, à la fois image et symbole de l'évolution positiviste de l'humanité.

Abstract: Exhibitions, museums, pavilions, artist ateliers, apartments and collectors'villas: exposition runs like a red thread through Le Corbusier's work. Man's relationship to art is a fundamental element of architectural dispositifs. Art influences his vision of society as a whole, and museums are central to his major urban plans, from the ziggurat of the Musée Mondial in Geneva, to the museums in Ahmadabad, Tokyo or Chandigarh, to projects he realized in the late 1960s, such as the Museum of the 20th Century in Nanterre. The evolution of museum design between 1929 and 1965 and of the concepts Le Corbusier developed for the different exhibitions of his own ouvre are in keeping with his way of understanding the relationship to works of art, whether by the artist, a knowledgeable public or those encountering art for the first time. The sketches for the different museums and pavilions retrace this evolution. Karel Teige's critique of the Mundaneum project assumes a key role in the transformation of the museum concept that occurred between the Musée Mondial of 1929 and Le Corbusier's first designs for a Museum with Unlimited Growth in 1930. The architecture and the place for art in society are no longer determined by the use of a form but through a functional mechanism. Growth is understood as an image of the positive evolution of mankind. This seminal change is a key to the later projects.

Mots clés: musée, exposition, fonctionnalisme.

Keywords: museum, exhibition, functionalism. 
Ateliers d'artistes, appartements et villas de collectionneurs, pavillons, scénographies et musées : l'exposition est un fil rouge de l'œuvre de Le Corbusier. Le rapport que l'homme entretient à l'œuvre d'art et les modalités de ce rapport sont des éléments fondateurs de son architecture et occupent une position primordiale dans sa vision de la ville. Les musées sont des pièces incontournables des ses grands plans d'urbanisme ou sont investis de rôles majeurs. De la ziggourat du Musée mondial en 1929 au projet du musée à croissance illimitée au début des années 1930 avec ses variantes successives, du plan de reconstruction de Saint-Dié à la construction des musées d'Ahmedabad, de Tokyo et de Chandigarh après la Seconde Guerre mondiale, jusqu'aux projets des années 1960, avec le Centre d'Art international à Erlenbach ou le Musée du XX ${ }^{\mathrm{e}}$ siècle pour Nanterre ${ }^{1}$, l'évolution des projets de musées entre 1929 et 1965 et des concepts des différentes expositions qu'il organise ou qui lui sont consacrées est parallèle à celle de sa manière d'envisager le rapport à l'œuvre, que ce soit celui de l'artiste, du spectateur initié ou du novice. Les esquisses préparatoires des différents projets de musées et de pavillons d'expositions retracent cette évolution. La critique du projet du Mundaneum de Karel Teige assume un rôle clé dans la transformation décisive du concept du musée qui a lieu entre le Musée Mondial en 1929 et le premier projet de Le Corbusier pour le Musée à croissance illimité en 1930. L'architecture et la relation à l'œuvre d'art ne sont plus déterminées par le recours à une forme, mais par un mécanisme fonctionnel. La croissance est l'image de l'évolution positiviste de l'humanité. Ce changement séminal est décisif pour les projets futurs.

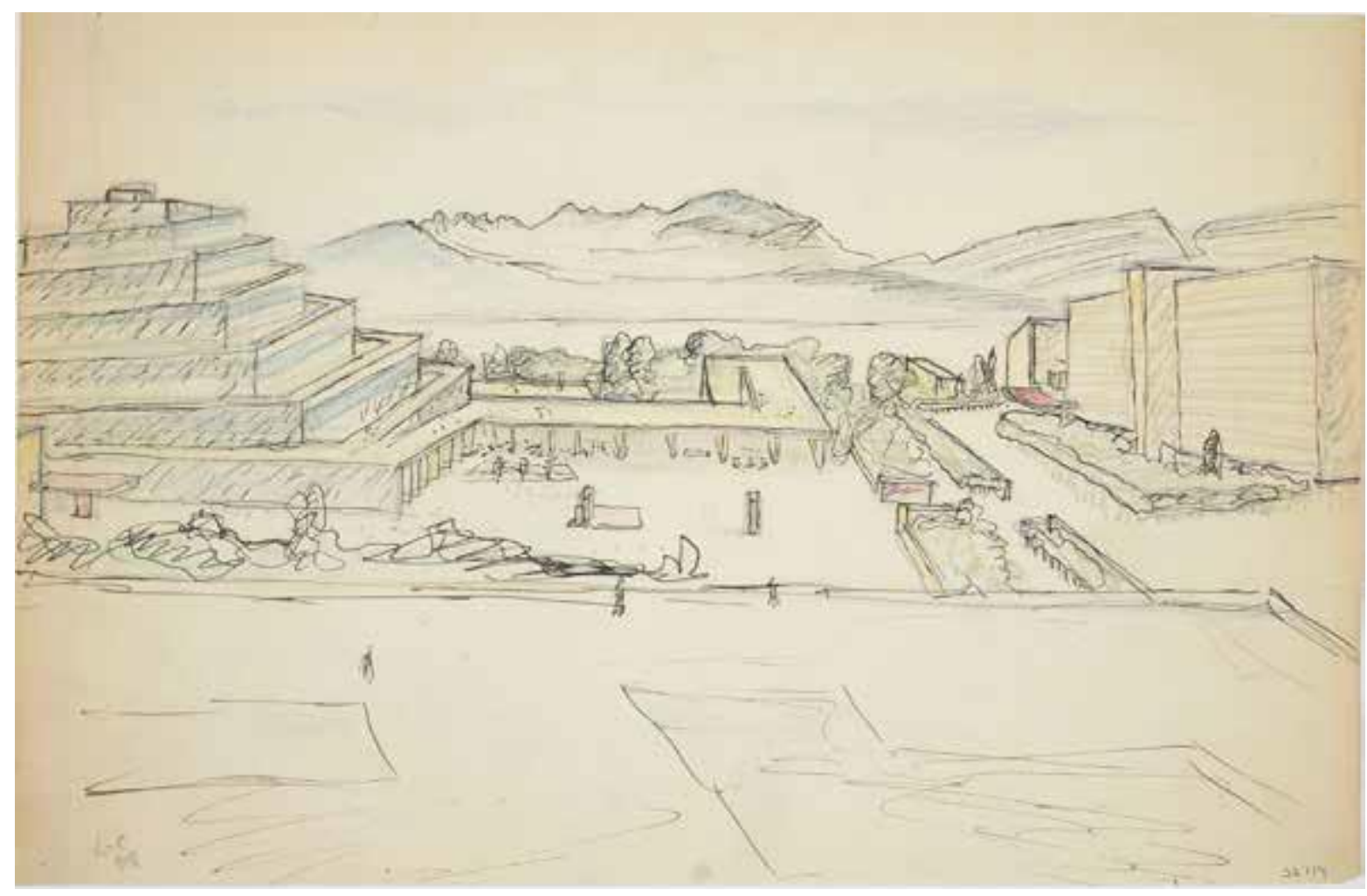

1. Le Corbusier et Pierre Jeanneret, Mundaneum, perspective de Le Corbusier montrant le panorama vers le lac Léman et le Mont-Blanc, FLC 32114.

\footnotetext{
${ }^{1}$ Sur les musées, voir Mory, Pascal : “Le Corbusier and the Syndrome of the Museum”. In Cohen, Jean-Louis ; Ahrenberg, Staffan (dir.), Le Corbusier's Secret Laboratory. From Painting to Architecture, Ostfildern : Hatje Cantz, 2013. pp. $256-281$.
} 


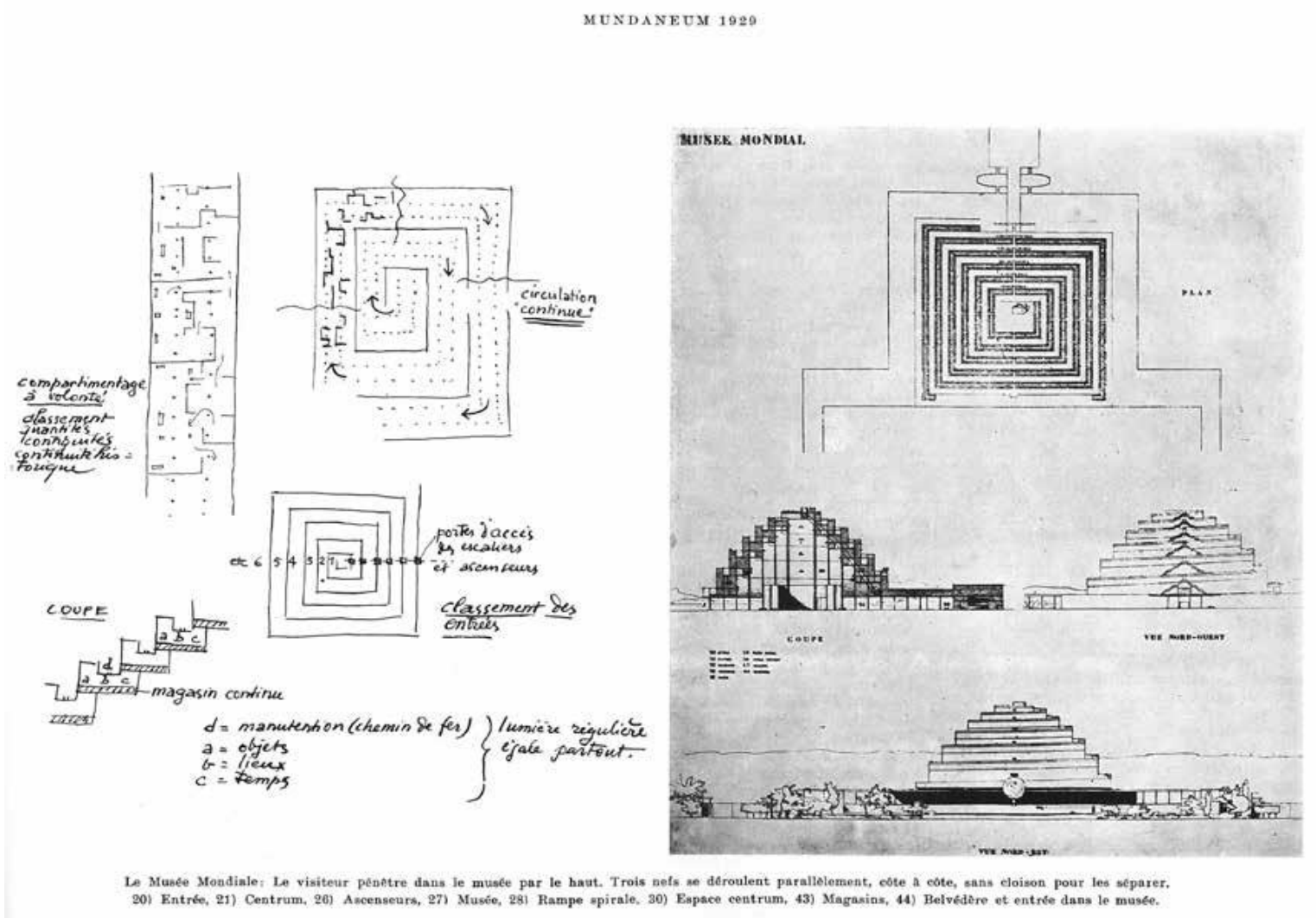

2. Le Corbusier et Pierre Jeanneret, Mundaneum, le Musée mondial, schéma montrant le fonctionnement de la circulation en spirale descendante et des trois nefs destinées aux objets (a), lieux (b) et temps (c) et le chemin de fer qui dessert le magasin continu (d), In Boesiger, Willy ; Storonov, Oscar (dir.) : Le Corbusier et Pierre Jeanneret, OEuvre complète. 1910-1929. Zurich : Gisberger, 1937. Vol. 1, p. 193.

\section{Le Musée mondial, 1928-1929, le musée comme monument à la culture}

Le premier musée dessiné par Le Corbusier et Pierre Jeanneret en 1928-1929 est le Musée mondial ou " musée de la connaissance humaine" ${ }^{2}$. Le programme, qui dépasse largement celui d'un musée d'art au sens habituel, est le lieu symbolique majeur de la Cité mondiale, le Mundaneum. Ce projet de créer une structure supranationale dédiée à la paix porte la marque des utopies universalistes de la fin du XIX $\mathrm{X}^{\mathrm{e}}$ siècle. Son ambition était de rassembler toutes les structures associatives existantes et de les intégrer dans un grand centre organisé autour de pôles culturels : la bibliothèque, l'université et, occupant le centre, le musée. Blaise Cendrars $^{3}$ avait organisé la rencontre de Le Corbusier avec Paul Otlet, figure visionnaire du mouvement pacifiste et instigateur du projet en 1927, au moment du concours pour le siège du Palais des Nations. Otlet avait établi dès les années 1910 un programme complet avec un organigramme détaillé de la Cité mondiale et du Mundaneum, un musée à visée universaliste et à haute charge symbolique qui aurait témoigné de l'essence des civilisations humaines.

\footnotetext{
${ }^{2}$ Voir Courtiau, Catherine : "La Cité internationale" et Gresleri, Giuliano : "Le Mundaneum. Lecture d'un projet". In Charollais, Isabelle ; Ducret, André (dir.). Le Corbusier à Genève 1922-1932. Projets et réalisations, catalogue d'exposition, Lausanne : Payot, 1987. pp. 53-69 et pp. 70-78. Voir aussi Gresleri, Giuliano : “ Mundaneum ”. In Lucan, Jacques (dir.), Le Corbusier, une encyclopédie. Paris : Éditions du Centre Pompidou, 1987. pp. 261-293.

${ }^{3}$ Blaise Cendrars est né à la Chaux-de-Fonds en 1887, la même année que Le Corbusier.
} 
Le Corbusier connaissait bien le site, une pente douce inclinée vers le lac Léman avec un panorama magnifique sur le mont Blanc et les Alpes d'un côté et le Jura de l'autre. Sur cet emplacement exceptionnel, la dimension paysagère est mise au cœur du dispositif urbain et architectural. La beauté du paysage, magnifiée par une scénographie grandiose, doit être garante de l'image universaliste que les institutions cherchent à se donner. Le Musée mondial est disposé à 45 degrés par rapport aux points cardinaux, suivant la trame du plan directeur de la Cité mondiale, il est ainsi adossé contre le Jura et orienté vers le lac, dans l'axe du mont Blanc. Sa forme monumentale marquée en fait le pivot de la composition de la Cité mondiale, l'élément d'ancrage dans le paysage lémanique. Ce paysage sert de coulisse à un parcours initiatique divisé en deux temps. Le visiteur doit entreprendre la longue ascension la pyramide en empruntant la rampe panoramique avant de pénétrer dans le musée par le haut. La rampe, longue de plus de 2,5 km implique une ascension d'au moins une demi-heure couronnée par la terrasse panoramique. Elle est l'instrument du conditionnement physique et mental nécessaire à la visite du musée, en créant à la fois un espace et un temps de transition pour le visiteur. Une fois à l'intérieur, il entame la descente de la spirale en suivant le parcours initiatique qui se déroule à travers les trois nefs contiguës présentant “L’homme dans le temps et dans le lieu. Exactement l'œuvre humaine reportée à l'époque de sa création et dans les lieux qui l'ont vu naître. L'œuvre. Le temps. Le lieu. ${ }^{4}$ ", une transposition littérale du programme de Paul Otlet. Les bords de la spirale sont fermés par des parois, la muséographie est basée sur l'organisation en trois nefs parallèles. Les schémas publiés dans l'Guvre complète montrent que le parcours à l'intérieur de la spirale n'était pourtant pas exclusivement linéaire. Les nefs sont matérialisées par des poteaux et le parcours est organisé par des cimaises librement disposées qui permettent de créer des ensembles et des espaces de dimensions variées selon les besoins. L'attention du spectateur est focalisée sur l'exposition, aucune ouverture ne vient le distraire de la contemplation des œuvres et du parcours de l'exposition : seules subsistent les œuvres et le fil narratif de l'exposition. Son parcours terminé, il sort sur l'esplanade de la Cité après avoir traversé le Sacrarium et se trouve devant le Globe, maquette du globe terrestre dans laquelle il peut entrer pour contempler la voûte céleste.

Le Corbusier restitue mieux que personne le lyrisme du projet : “ $\grave{A}$ chaque tournant, un horizon neuf; $\grave{a}$ chaque spirale une vue plus dégagée. Le site grandit à mesure. Au sommet, le site est là tout entier, panoramique : les Alpes les plus altières, le lac le plus suave, la ville tapie au fond; au pied de ses rochers horizontaux, le Rhône, ce grand fleuve du monde, qui s'enfonce vers la mer... Alors il pénètre dans le musée par le haut. Il voit les tableaux de la gestation du monde : les nébuleuses qui se forment en soleil ; le mécanisme des planètes, la séparation de l'air, de l'eau et de la terre. [... $]^{5}$ " Le parcours initiatique doit provoquer l'empathie du spectateur et accompagner un cheminement intérieur. Giuliano Gresleri a analysé l'importance et la signification de ce parcours qui, contrairement aux ziggourats antiques ou à la tour de Babel, ne suit pas un mouvement ascendant, allant vers le divin. Il note que : "Malgré l'émotion que Le Corbusier réussit à nous transmettre, la descente “impossible” - qui aurait duré trois à quatre heures échappait ainsi à l'idée, chère à la tradition de l'expressionnisme, d'un itinéraire mystique vers le sacré, d'une montagne ou d'un escalier à gravir. Par contre, elle devenait le chemin de la rationalité, du savoir, tous deux saisis à travers l'histoire même de l'homme. ${ }^{6}$ "

\footnotetext{
${ }^{4}$ Le Corbusier : “ Mundaneum, 1929 ”. In Boesiger, Willy ; Storonov, Oscar (dir.) : Le Corbusier et Pierre Jeanneret. Euvre 1929-1910 .complète. Zurich : Gisberger, 1937. $2^{\mathrm{e}}$ édition. Vol. 1. p. 194.

${ }^{5}$ Le Corbusier, cité par Gresleri, Giuliano : “Le Mundaneum. Lecture d'un projet”. In Charollais, Isabelle; Ducret, André (dir.) : Le Corbusier à Genève 1922-1932. Projets et réalisations, catalogue d'exposition. Lausanne : Payot, 1987. pp. 70-78, citation page 76 .

${ }^{6}$ Gresleri, Giuliano : “Le Mundaneum. Lecture d'un projet”. In Charollais, Isabelle; Ducret, André (dir.) : Le Corbusier à Genève 1922-1932. Projets et réalisations, catalogue d'exposition. Lausanne : Payot, 1987, p. 77.
} 


\section{La critique de Karel Teige}

La monumentalité affirmée du projet déclenche incompréhension et critiques dans le camp même des Modernes, en particulier de la part de Karel Teige ${ }^{7}$. Dans les pages de Stavba ${ }^{8}$, il publie un long article composé d'une description détaillée du projet suivi d'une critique attentive. Dans ce fameux texte dans lequel il pointe l'absence de légitimité du recours à des formes historiques et le "romantisme " inhérent à l'emploi d'une "section d'or ", il se livre en premier lieu à une analyse sévère du programme: "Where do the roots of the non-modern, and in fact archaic character of Le Corbusier's Mundaneum lie? To what should we attribute this architectural error and delusion? Actually, in our view, the first root of this misconception of the program lies in the program, the idea and the theory of the Mundaneum. This idea is not alive, it doesn't originate from a vibrant, felt need; it's the fruit of the abstract and rarified speculation of intellectual coteries within the League of Nations. "Il critique ensuite durement les illusions et la naïveté de Otlet et d'Andersen, avant de revenir sur le terrain de l'architecture: "It is an oft-repeated and confirmed experience that the architectural investigation of problems and programs which are ideologically unclear, falsely stated, or moribund, cannot produce works of elemenatary clarity and purity. " Puis il rappelle l'indissociable relation qui, à ses yeux, unit l'architecture moderne au fonctionnalisme: "Modern architecture was born not from abstract speculation, but from actual need, from the dictates of life, not the patronage of some academy or official group. Real need furnished programs: factories, bridges, railway stations, offices, housing for workers, schools, hospitals, hotels and apartments; from a fundamental understanding and shaping of these problems pure modern architecture was born. "Et il conclut sans appel sur l'échec programmé de toute autre tentative: "Examples of concrete and utilitarian architecture, as well as omens of a new metaphysical, monumental architecture both show clearly that, at the present time, architecture will fail in so far as it is not dictated by the actual needs of social and economic life. The only aim and scope of modern architecture is the scientific solution of exact tasks of rational construction."

Le Corbusier dans sa tout aussi fameuse "Défense de l'architecture" " répond point par point aux attaques sur l'emploi de la forme de la pyramide, sur celui du nombre d'or, se saisissant magistralement de l'occasion pour asseoir les bases de son discours théorique, se refusant à réduire l'architecture à la seule expression d'une fonction et se ralliant au mot d'ordre "L'utile n'est pas le beau ". Par contre, à aucun moment, il n'argumente sur la question du programme, ni ne cherche à légitimer sa validité ou son actualité, il reste muet sur ce point. Il est toujours en pourparlers avec Paul Otlet, à qui il remettra la dernière étude pour la Cité mondiale sur la rive gauche d'Anvers en 1933 et ne tient sans doute pas à remettre en question les positions d'un client. Mais il est ici possible de faire l'hypothèse que sur ce point, la critique de Teige a atteint son but: malgré quelques ressemblances formelles évidentes, le projet du Musée d'art contemporain pour Paris sur lequel il travaille dès l'année suivante montre en effet un spectaculaire retournement des paradigmes et des choix projectuels qui avaient présidé à la composition du Musée mondial.

\footnotetext{
${ }^{7}$ Sur cette question voir Cohen, Jean-Louis : "Le Corbusier et les théories de l'avant-garde soviétique ". In Cohen, JeanLouis : Le Corbusier et la mystique de l'URSS. Bruxelles: Mardaga, 1987. pp. 142-150 et Reichlin, Bruno: "Solution élégante ”. In Lucan, Jacques (dir.) : Le Corbusier, une encyclopédie. Paris : Édition du Centre Pompidou, 1987. pp. 369-377. ${ }^{8}$ Teige, Karel “Mundaneum ”. In Stavba. Avril 1929. ํ1․ pp. 145-155. La traduction anglaise parue dans Opposition. Octobre 1974. $\mathrm{N}^{\circ}$ 4. pp. 80-90.

${ }^{9}$ Le Corbusier : “Défense de l'architecture”. In L'Architecture d'aujourd'hui. 1933. № 10. pp. 38-61. L'article initialement écrit en 1929 pour la revue Stavba a été publié en tchèque dans Musaion. 1931, № 2, pp. 27-52. Sur l'importance de ce texte voir Reichlin, Bruno: "Solution élégante”. In Lucan, Jacques (dir.) : Le Corbusier, une encyclopédie. Paris : Édition du Centre Pompidou, 1987. pp. 369-377.
} 


\section{Le Musée d'art contemporain, 1930-1931, la première occurrence du Musée à croissance illimitée}

Le projet pour le Musée d'art contemporain prend la forme d'un envoi adressé à une figure tutélaire de l'avant-garde artistique, Christian Zervos, directeur des Cahiers d'art, et destiné à être publié dans cette revue $^{10}$. C'est un exercice que l'architecte se pose en l'absence d'une commande concrète, pratique à laquelle il recourt souvent. Il s'inscrit dans le contexte français de l'époque et dans une réflexion sur le rôle des musées qui fait écho au manque d'engagement des institutions face aux avant-gardes, peu présentes dans les collections publiques, et leur manque de réactivité par rapport aux débats artistiques contemporains. Auguste Perret avait publié en 1929 dans la revue Mouseion "11, l'organe de l'Office international des musées, un texte intitulé "Le musée moderne " exposant sa conception d'un "édifice qui serait à la fois un lieu de délectation et de fête et un lieu d'étude ", une rotonde et un espace central destinés à l'exposition des chefs d'œuvre et, ouvertes sur cet espace central, des "galeries de classement et d'études ", disposées comme un magasin de réserve, qui permettrait l'accès au visiteur. L'article, illustré d'un plan et d'une perspective à main levée, traite principalement de questions techniques, des avantages du béton, de la justesse de l'éclairage et de l'adaptation aux différentes œuvres exposées, peinture ou sculpture, ou encore au climat, mais n'engage pas vraiment un débat de fond et n'est pas destiné à la présentation concrète d'un projet. Le Corbusier de son côté cherche à asseoir sa position comme interlocuteur incontournable sur la question des programmes muséaux et propose un modèle prêt à l'emploi, "brevetable ", et surtout reproductible. Son choix de le publier dans les Cahiers d'art, donc dans une revue d'art, n'est pas un hasard. Sigfried Giedion y tient régulièrement une chronique sélective sur l'architecture et y a publié ses projets. L'architecte côtoie dans les pages de la revue les figures marquantes de l'avant-garde, bénéficiant ainsi ipso facto de leur caution.

\footnotetext{
${ }^{10}$ Le Corbusier et Jeanneret, Pierre : “Pour la création à Paris d'un musée des artistes vivants”, In Cahiers d'art. 1931. №1, p. 9.

${ }^{11}$ Perret, Auguste: “Le musée moderne ”. In Mouseion. Décembre 1929. № 9. pp. 225-235. La revue est créée en 1927, un an après la fondation de Office international des musées, ancêtre de l'ICOM, organisme dépendant de la Société des nations. La revue dont le siège est à Paris est très proche de l'OIM et joue un rôle pionnier dans les débats sur la muséographie, abordant des questions allant de la conservation des œuvres, au patrimoine monumental, au développement de nouveaux programmes comme les musées paysans ou les musées de plein-air, à l'architecture des musées et des expositions. Voir le résumé de la thèse de Marie Caillot : http://theses.enc.sorbonne.fr/2011/caillot.
} 

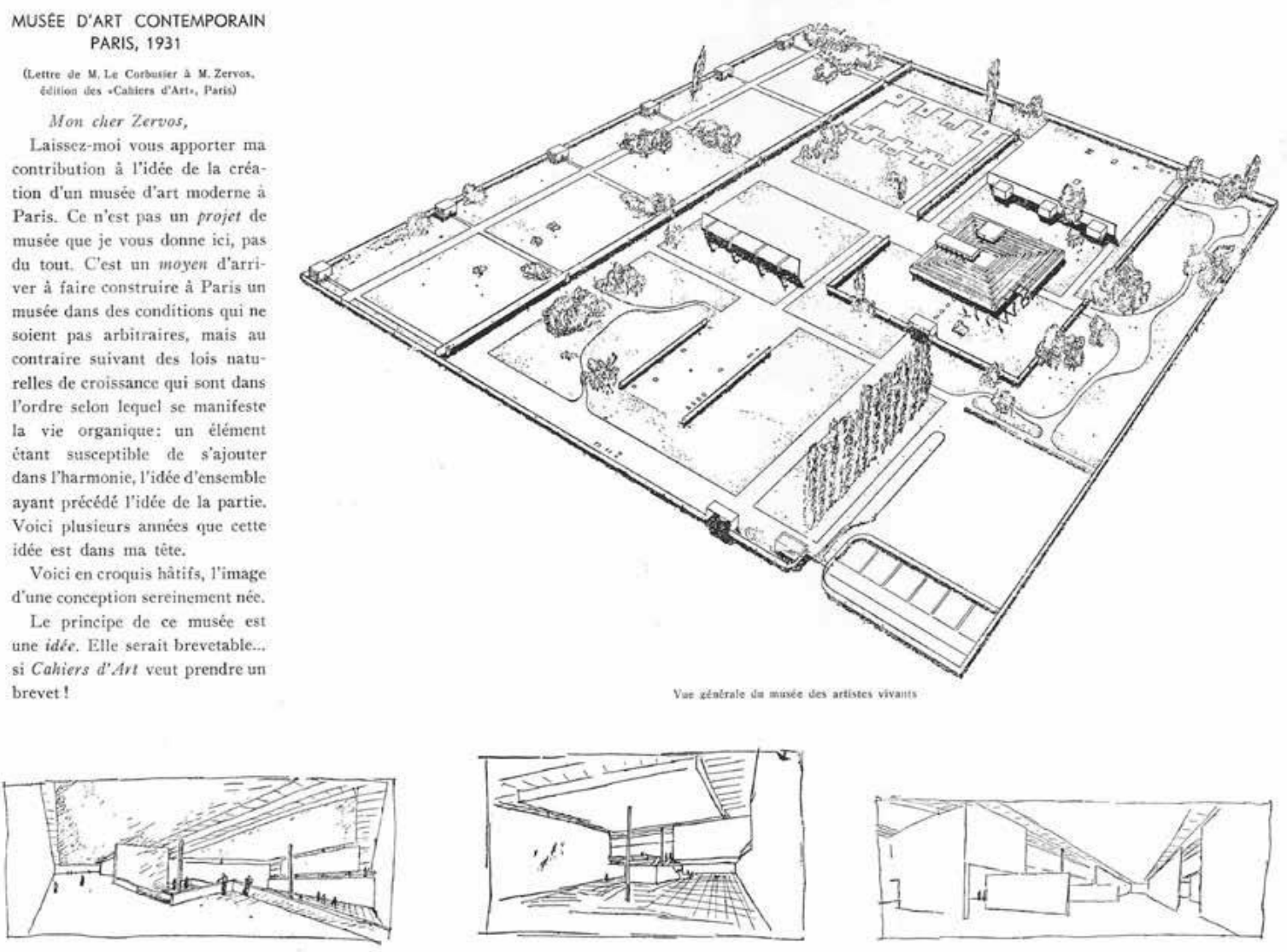

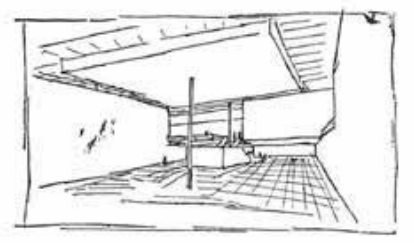

Asoect de la premierre walle; so land, en haut, on apercoit

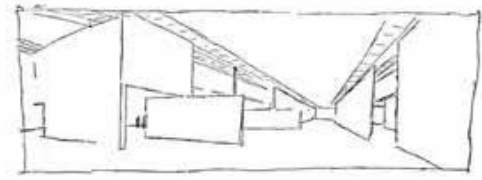

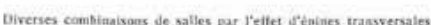

Aspect de la premitere salle entourte de ta premilite nef de la spice

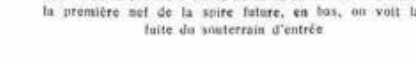

3. Le Corbusier et Pierre Jeanneret, Musée d'art contemporain, Paris, 1930, perspective aérienne. In Boesiger, Willy (dir.) : Le Corbusier et Pierre Jeanneret, OEuvre complète. 1929-1934. Zurich : Gisberger, 1935. vol. 2. p. 72.
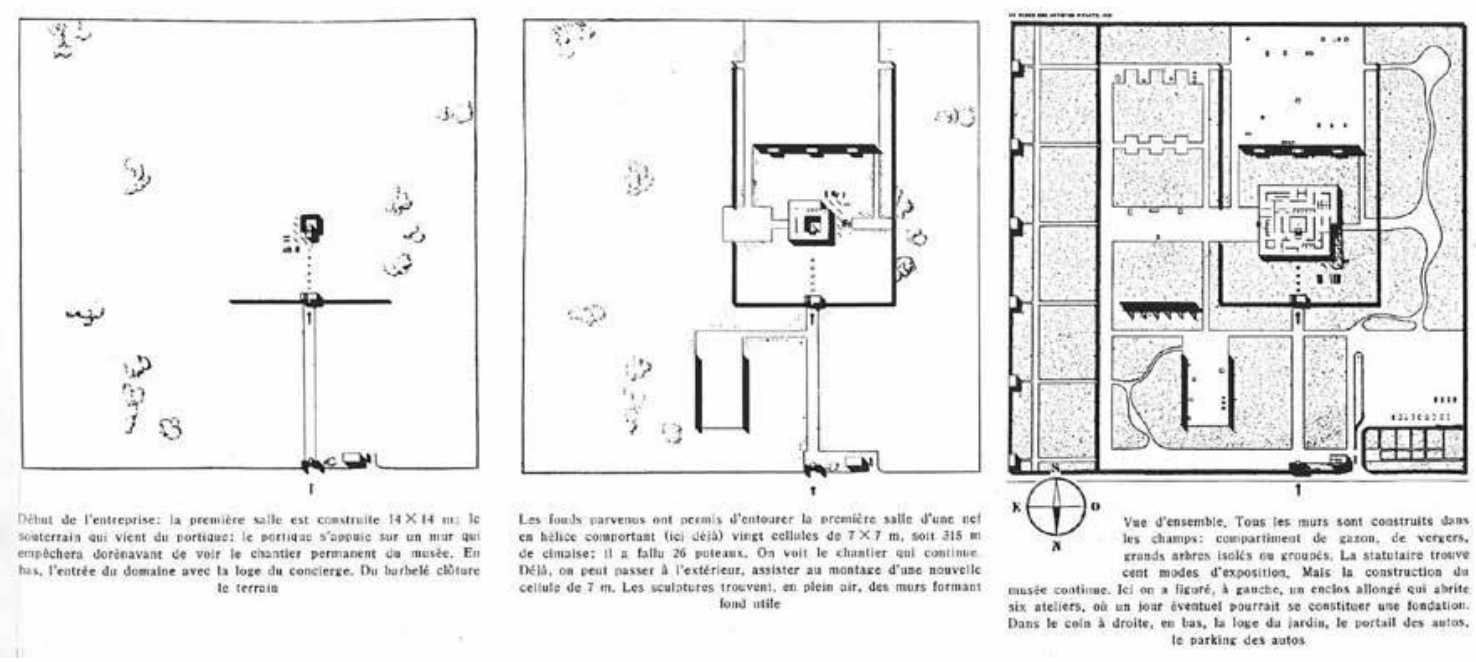

4. Le Corbusier et Pierre Jeanneret, Musée d'art contemporain, Paris, 1930, principe de croissance. In Boesiger, Willy (dir.): Le Corbusier et Pierre Jeanneret, OEuvre complète. 1929-1934. Zurich : Gisberger, 1935. vol. 2. p. 73. 


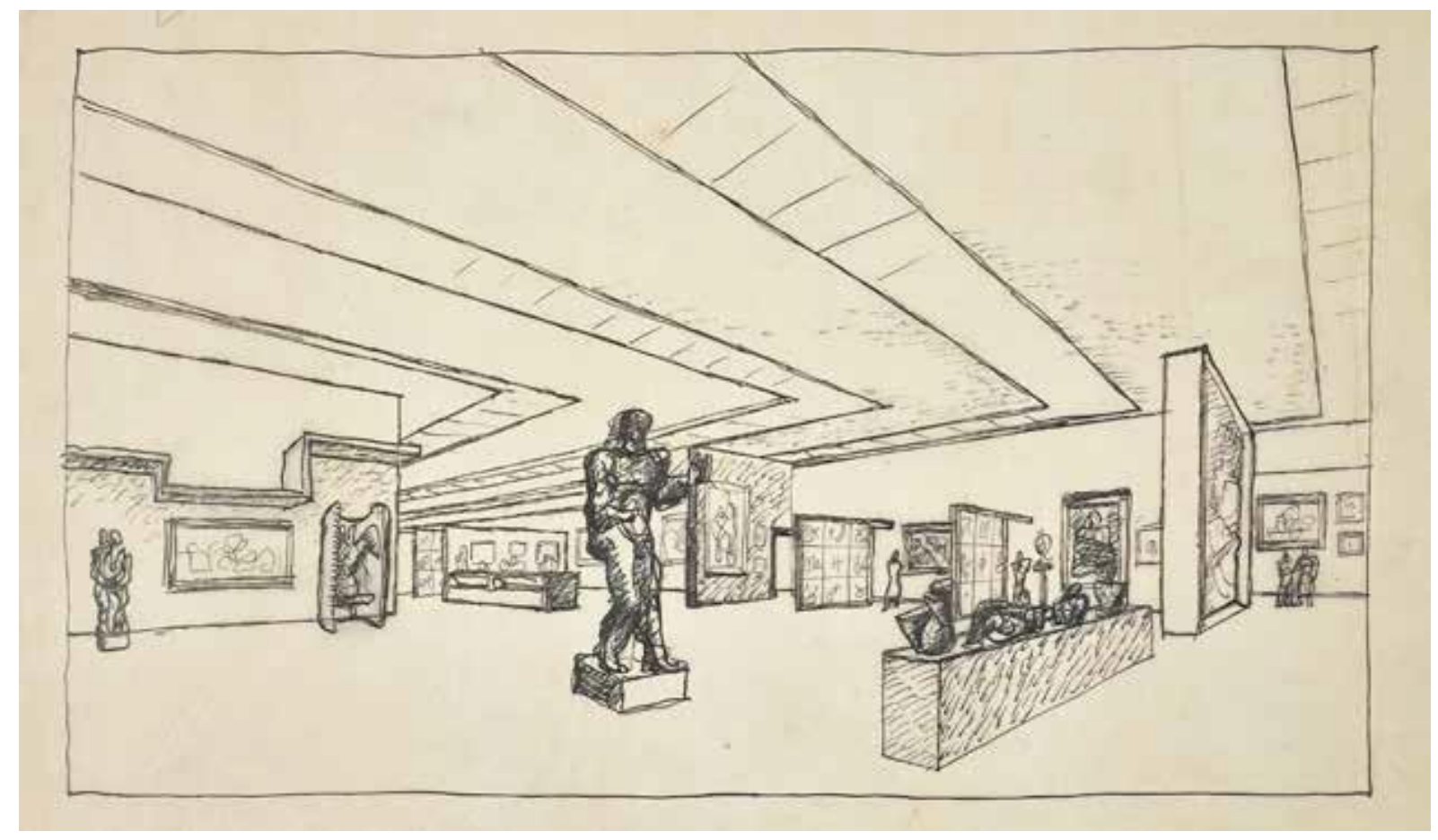

5. Le Corbusier et Pierre Jeanneret, Musée d'art contemporain, Paris, 1930, perspective, FLC 30037.

Au premier coup d'œil, le Musée d'art contemporain avec son iconique spirale carrée semble directement inspiré du projet du Mundaneum; pourtant le projet et les dessins préparatoires montrent une césure spectaculaire. La ziggourat a disparu, le parcours est désormais organisé dans un seul plan, la forme de la spirale maintenant aplatie autorisant un accroissement lent et continu de la surface du musée impossible dans le projet de 1929. C'est la première occurrence du musée à croissance illimitée. Le Corbusier cherche à le promouvoir en tant qu'idée et non en tant que projet, il écrit à Christian Zervos : “Ce n'est pas un projet que je vous donne ici, pas du tout. C'est un moyen d'arriver à faire construire à Paris un musée dans des conditions qui ne soient pas arbitraires, mais au contraire suivant les lois naturelles de croissance qui sont dans l'ordre selon lequel se manifeste la vie organique : un élément étant susceptible de s'ajouter dans l'harmonie, l'idée de l'ensemble ayant précédé l'idée de la partie. [...] Le principe de ce musée est une idée. Elle serait brevetable... ${ }^{12}$ " Mais un autre pas est franchi, le bâtiment, extensible, ne possède plus de forme fixée dans la durée: "Le musée n'a pas de façade; le visiteur ne verra jamais de façade; il ne verra que l'intérieur du musée. ${ }^{13}$ ". Le rapport au site, ville ou paysage, est mis à distance, voir indifférent: "Le musée s'élève dans quelque banlieue ou grande banlieue de Paris. Il s'élève au milieu d'un champ de pommes de terre ou de betteraves. Si le site est magnifique, tant mieux. S'il est laid et attristé de pignons de lotissements ou de cheminées d'usine, ça ne fait rien $[\ldots]^{14}$ ". Le mécanisme d'agrandissement du bâtiment implique un abandon de la façade en tant qu'enveloppe possédant un forme durable et qu'élément distinctif de l'image du bâtiment. La démonstration de ce non-conformisme programmatique se retrouve affiché jusque dans le détail de la clôture du terrain qui est prévue en barbelés. Le Corbusier cherche à se délester d'un ensemble de contraintes constructives, stylistiques et formelles pour se concentrer sur un principe fondateur qui guide la conception architecturale. La forme est la démonstration du principe directeur, ici le principe de croissance.

\footnotetext{
${ }^{12}$ Le Corbusier : "Musée d'art contemporain”, Paris, 1932. In Boesiger, Willy (dir.) : Le Corbusier et Pierre Jeanneret, Cuvre complète. 1929-1934. Zurich : Gisberger, 1935. Vol. 2. pp. 72-73.

${ }^{13}$ Ibid.

${ }^{14}$ Ibid.
} 
La publication ne fournit que peu d'informations sur les choix muséographiques, quelques perspectives à main levée donnent une idée de l'atmosphère intérieure, montrant la salle centrale et le système d'éclairage zénithal. C'est une machine nue qui est livrée prête à l'emploi. L'accès par le centre prévu pour le Mundaneum persiste, de même que la trame dimensionnelle, un carré central de $14 x 14 \mathrm{~m}$, qui s'agrandit par l'ajout de modules de 7x7m. Les cloisons-membranes prévues à l'intérieur peuvent être déplacées et laissent toute liberté au discours scénographique. Le parallélisme des trois nefs présentant corollairement les trois récits - objets lieux et temps - a disparu, et avec lui, l'hypothèse d'une narration simple de l'évolution. Les perspectives intérieures montrent une architecture qui se met en retrait, quelques plans abstraits qui servent de support aux œuvres et un système d'éclairage zénithal, la forme cherche à être neutre, le sens naît maintenant de la relation entre les œuvres elles-mêmes.

\section{Le Musée de la Ville et de l'État, 1934}

Le Corbusier et Pierre Jeanneret ont la possibilité de prendre part au concours pour la construction d'un bâtiment réunissant le Musée des artistes vivants, situé alors dans l'Orangerie du Palais du Luxembourg, et le Musée de la Ville, concours qui aboutira à la construction du Palais de Chaillot. Leur contribution présente certaines similarités avec le projet du Musée d'art contemporain, mais il reprend certains éléments de la scénographie muséale du Mundaneum. C'est une forme hybride qui inclut certains éléments des deux projets précédents. La forte pente du terrain et la densité du programme impliquent de nouveau le recours à un empilement des galeries. La parcelle est entièrement utilisée, et la question d'une extension ou d'un phasage ne se pose pas. Deux demi-spirales carrées, l'une s'évasant, l'autre se rétrécissant, sont collées dos à dos de manière à produire une structure en $\mathrm{H}$ ouverte d'un côté vers la Seine, de l'autre vers l'avenue du Président Wilson, exprimant ainsi visiblement la division entre les deux musées qui composent le programme. Le vide intérieur devient ainsi un espace public ouvert, profitant du site magnifique au centre de Paris. La scénographie urbaine est exposée avec des termes qui renvoient directement à une toile de fond plus paysagère qu'urbaine: "La promenade des visiteurs est nette comme une route de montagne ouvrant à gauche et à droite sur des vallées successives. Ce trajet direct constitue la visite touristique. Chaque vallée, c'est une section muséographique déterminée, mais chacune se dédouble et se triple en une galerie latérale d'étude (où sont des ouvres momentanément reconnues moins démonstratives que celles exposées dans la piste touristique) et une galerie intérieure de stocks (où chaque section muséographique installe son stock dans un étalage et une lumière déjà parfaitement suffisante). ${ }^{15}$ " Il convient de noter le dispositif de la galerie intérieure de stocks éclairée, prémisse d'une discussion sur l'accessibilité plus large des réserves pour un public spécialisé.

\footnotetext{
${ }^{15}$ Le Corbusier : “ Plans pour les musées de la Ville et de l'Etat à Paris ”, Paris, 1932. In Bill, Max (dir.) : Le Corbusier et Pierre Jeanneret, Euvre complète. 1934-1938. Zurich : Gisberger, 1953. Vol. 3. pp. 82-89.
} 


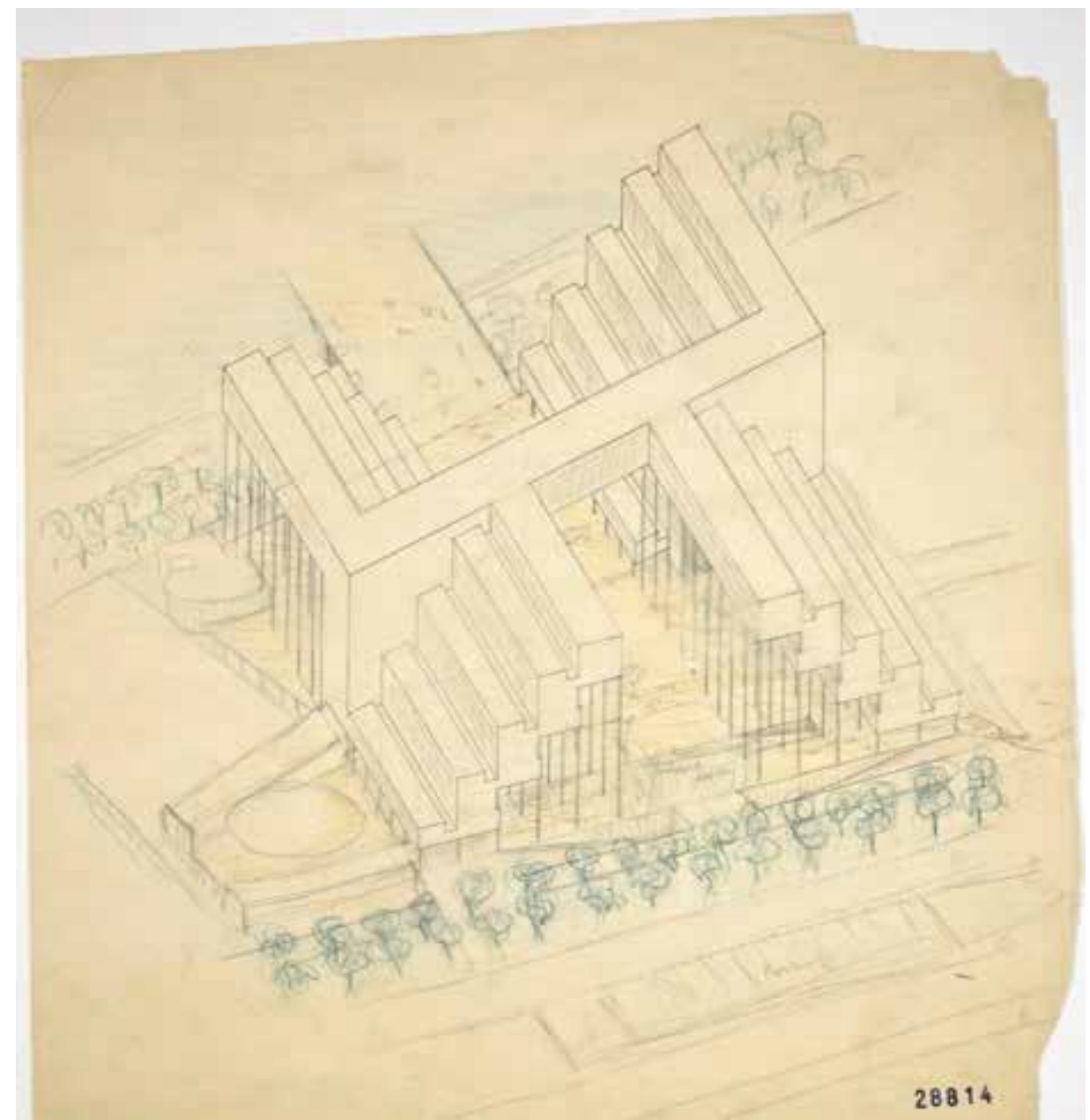

6. Le Corbusier et Pierre Jeanneret, Musée de la ville et de l'état, Paris, 1934, perspective, FLC 28814.

\section{Le projet $C$ pour l'exposition de 1937, le Centre d'esthétique contemporaine}

Les projets consécutifs réalisés en vue de l'Exposition internationale de Paris en 1937 sont le prochain laboratoire d'expérimentation pour les structures d'exposition. Après diverses tentatives infructueuses de construire un musée permanent, Le Corbusier et Jeanneret travaillent à partir de 1936 sur le projet C, une occurrence du musée à croissance illimitée dont ils définissent eux-mêmes le programme, une structure dont ils maitriseraient à la fois le contenant et le contenu. Cette situation privilégiée les conduit à étudier très en détail les relations et les interactions possibles entre les espaces générés par la spirale, la dynamique du parcours et les contenus. Les schémas FLC 705 et FLC 713 montrent la superposition de la trame constructive suivant les entraxes de 7 mètres et d'un parcours muséographique interdisciplinaire du type "musée de la connaissance ". Le parcours commence au centre par 1' " interdépendance des choses et de la culture " puis traverse l' " âge de la pierre " avant de traverser les différentes disciplines scientifiques - sociologie, éthique, philosophie -, puis les sciences physiques - biologie, astronomie -, continuant vers les inventions et les techniques, placées juste avant les mathématiques qui clôturent le parcours. Le plan FLC 675 montre la manière de concilier la flexibilité du dispositif spatial avec le contrôle du cheminement et de la progression dans l'exposition. Cette gestion d'un plan libéré de tout cloisonnement vertical fixe est désormais la constante de tous les projets ultérieurs. L'éclairage zénithal est le seul indicateur spatial qui impose certaines règles dans la façon de subdiviser l'intérieur. 


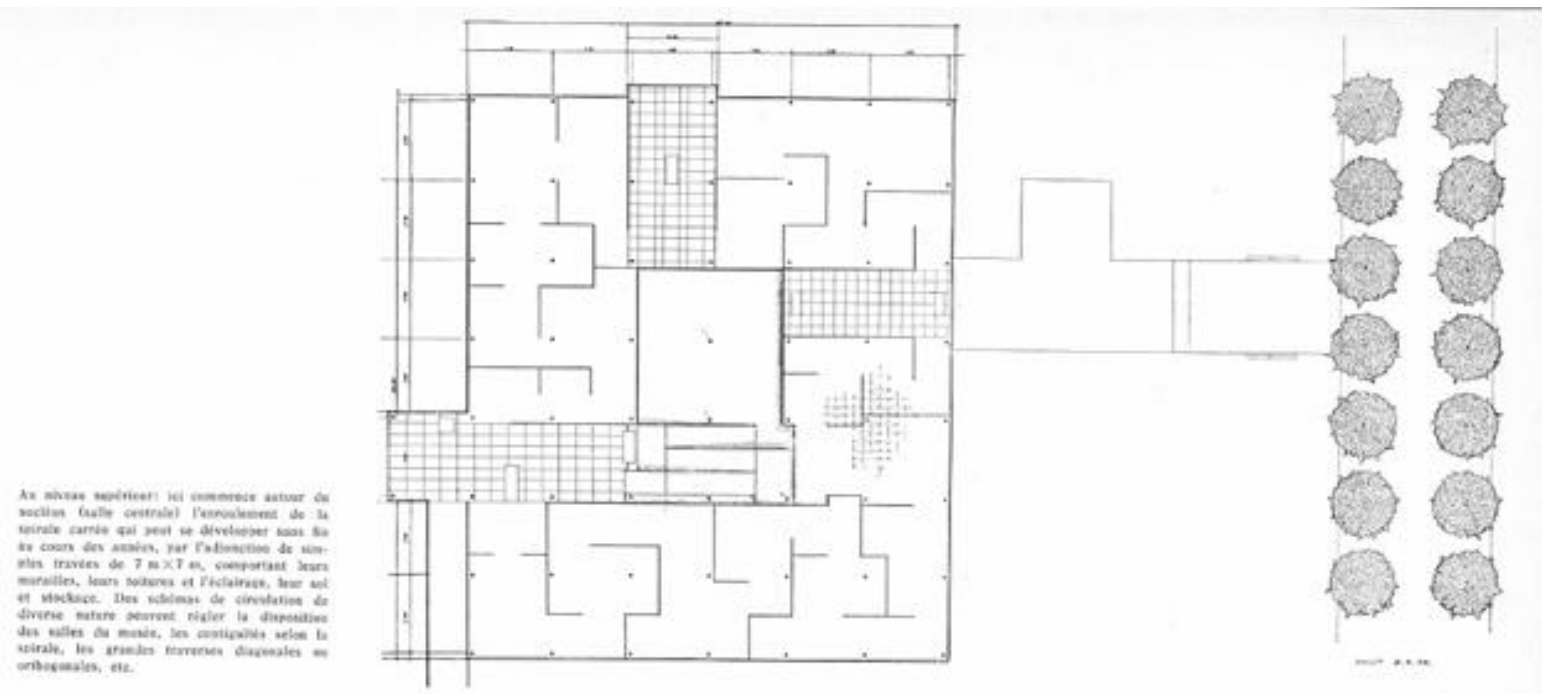

7. Le Corbusier et Pierre Jeanneret, Expo 37, Projet C, plan et élévation. In Boesiger, Willy (dir.) : Le Corbusier et Pierre Jeanneret, OEuvre complète. 1934-1938. Zurich : Gisberger, 1938. Vol. 3. p. 155.

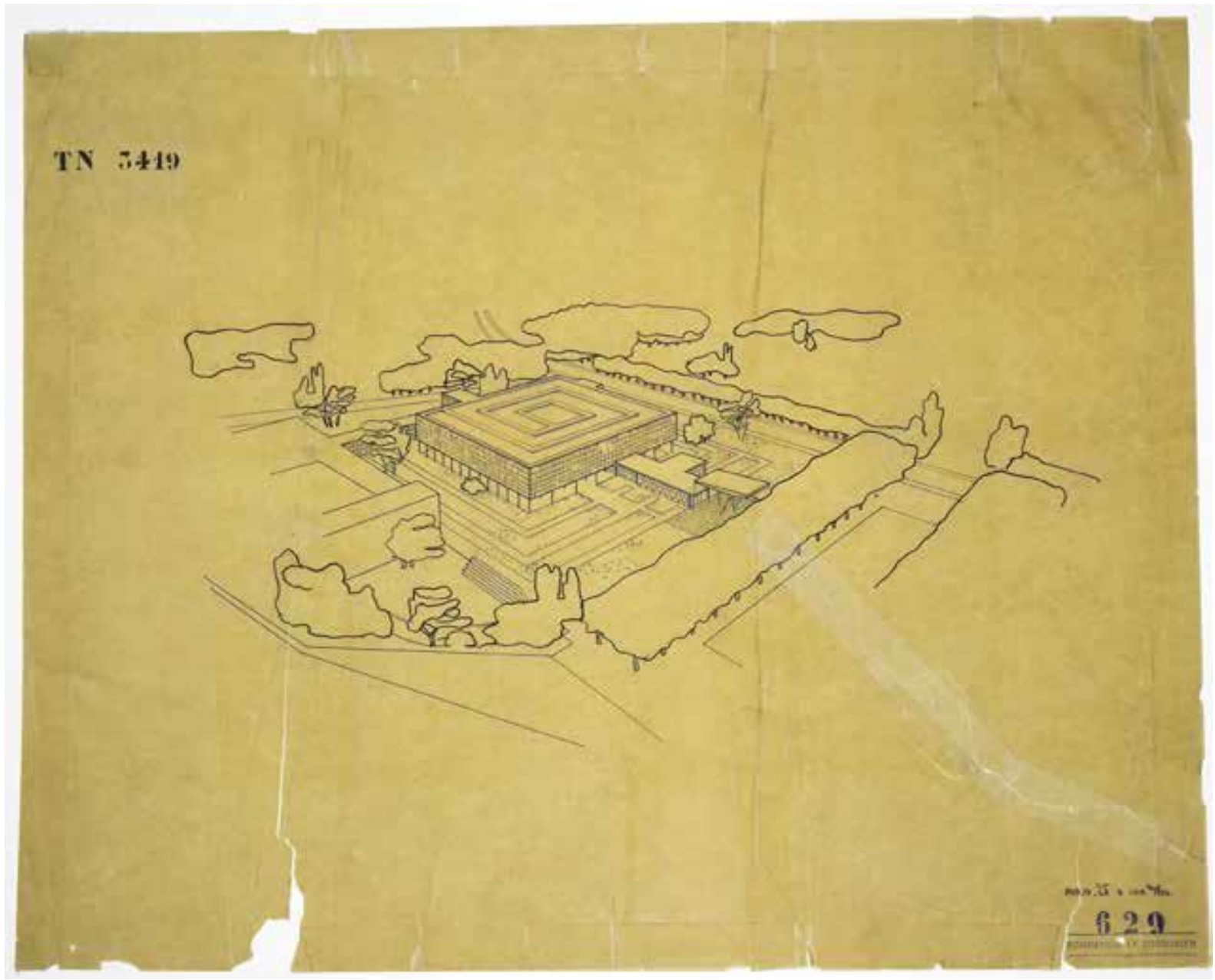

8. Le Corbusier et Pierre Jeanneret, Expo 37, Projet C, perspective à vol d'oiseau, FLC 626. 


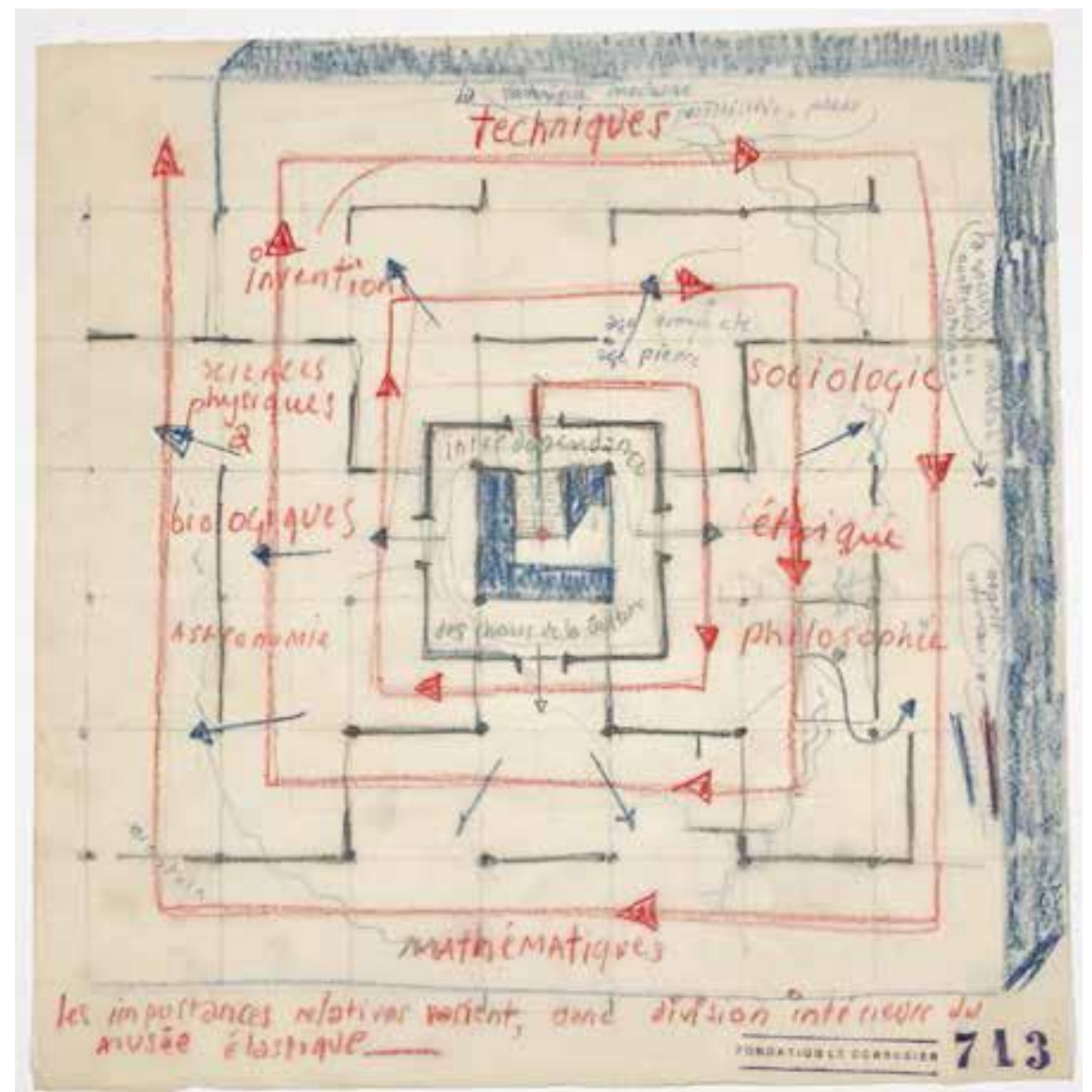

9. Le Corbusier et Pierre Jeanneret, Expo 37, Projet C, schéma de circulation, FLC 713.

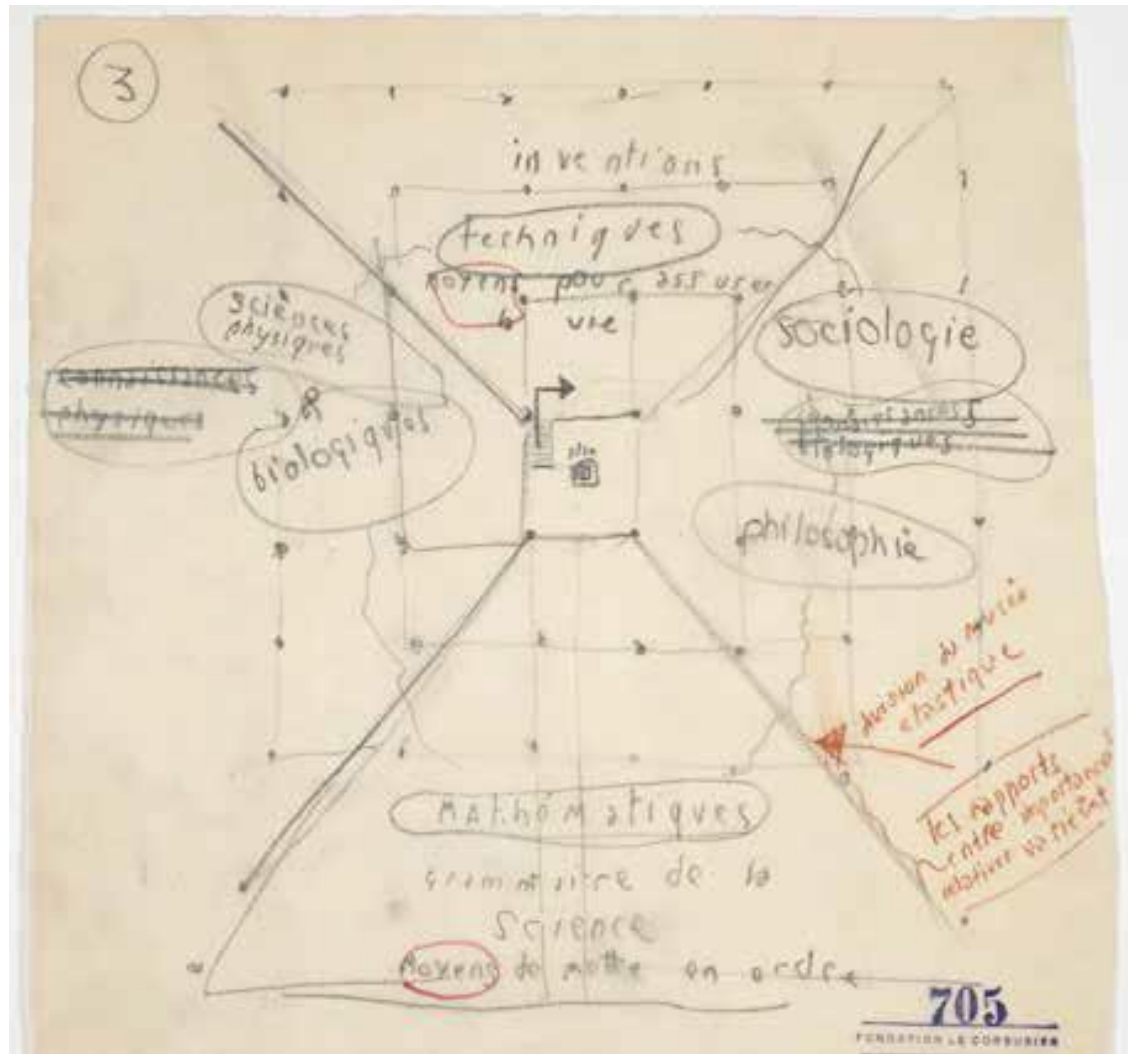

10. Le Corbusier et Pierre Jeanneret, Expo 37, Projet C, schéma d'organisation, FLC 705. 


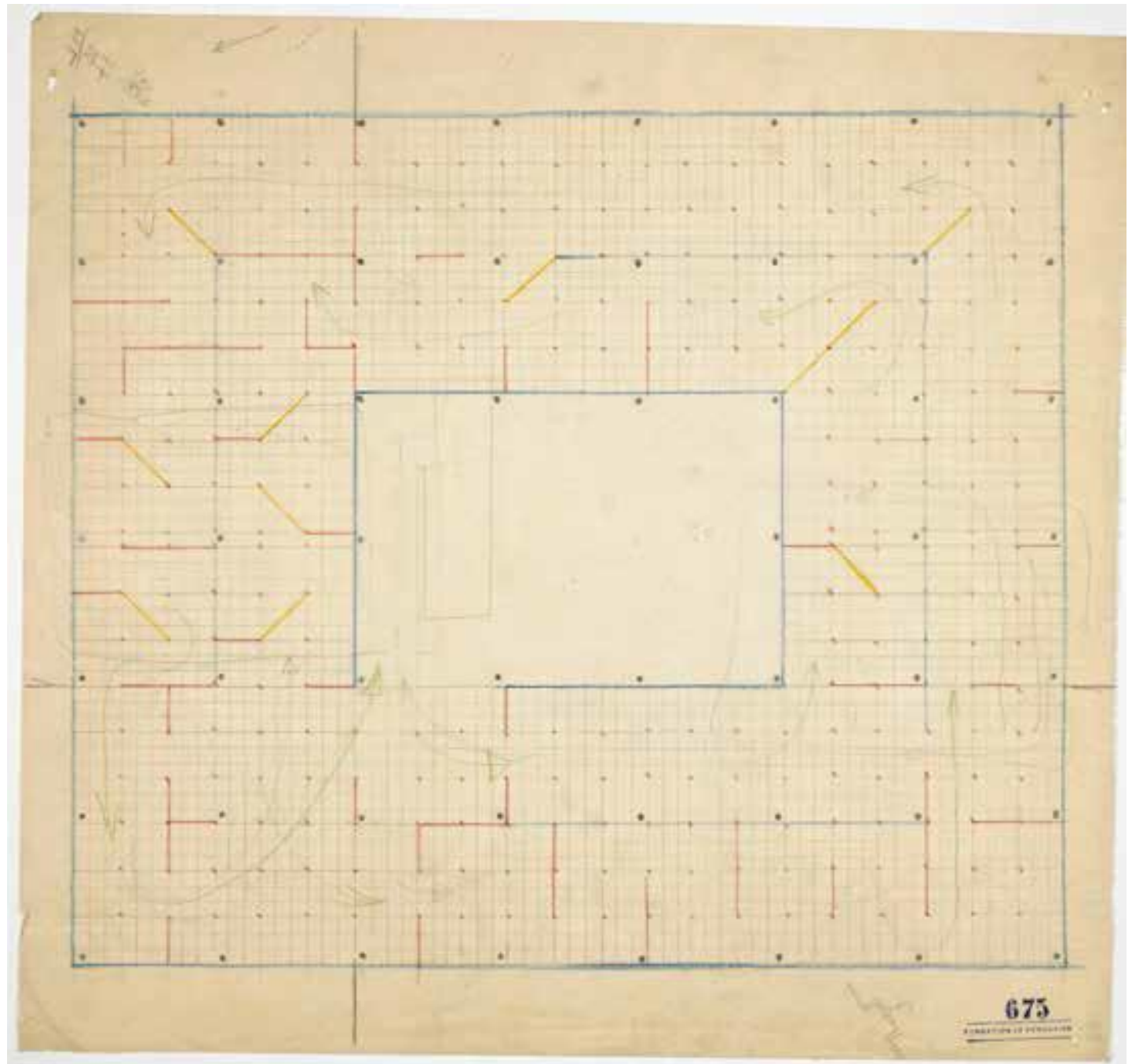

11. Le Corbusier et Pierre Jeanneret, Expo 37, Projet C, schéma d'organisation, FLC 675.

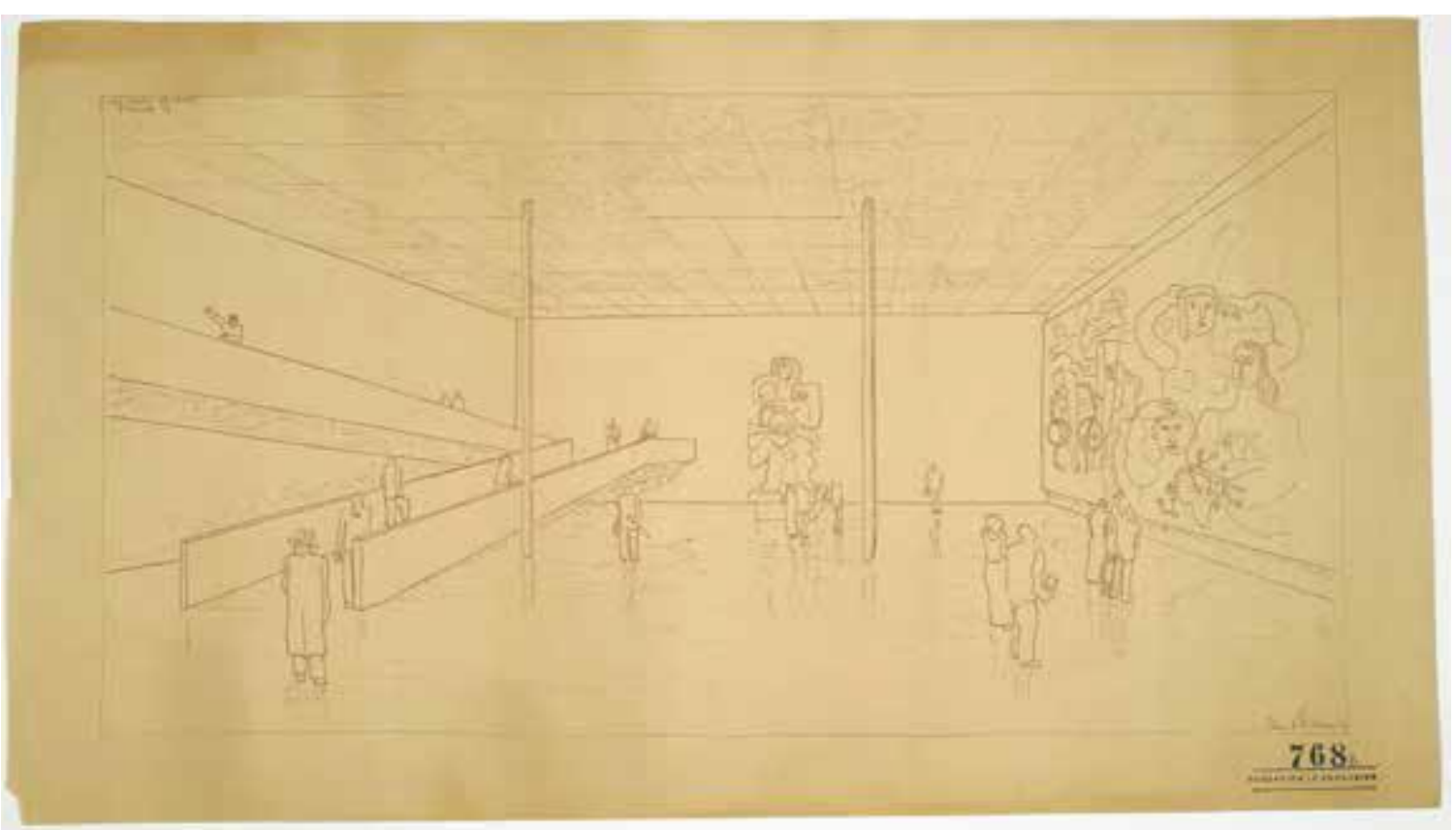

12. Le Corbusier et Pierre Jeanneret, Expo 37, Projet C, perspective, FLC 768 


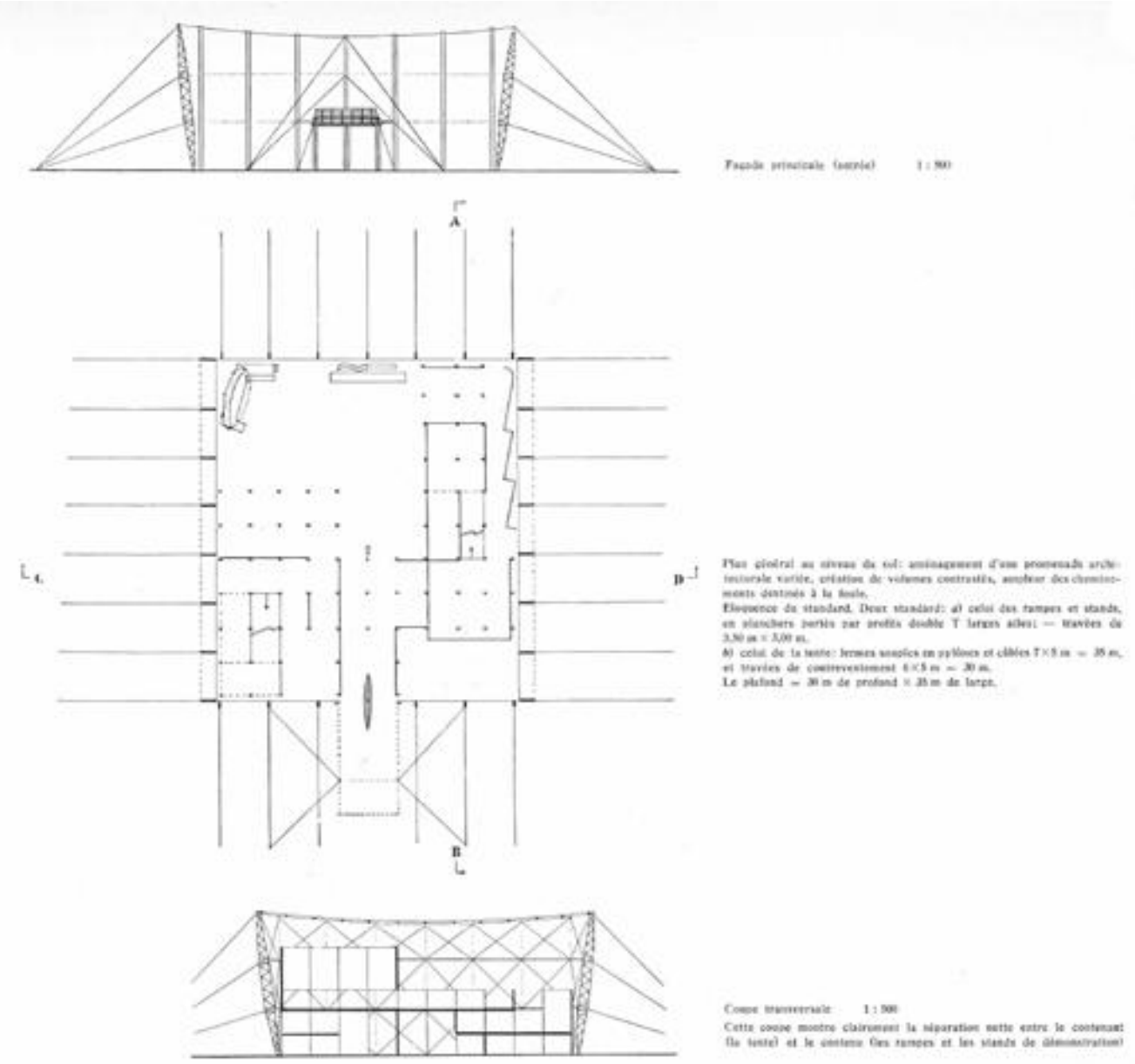

13. Le Corbusier et Pierre Jeanneret, Pavillon des Temps nouveaux, Expo 37, Projet D, élévation, plan et coupe. In Boesiger, Willy (dir.) : Le Corbusier et Pierre Jeanneret, OEuvre complète. 1934-1938. Zurich : Gisberger, 1939. Vol. 3. p. 160.Boesiger, Willy (dir.) : Le Corbusier et Pierre Jeanneret, OEuvre complète. 1934-1938. Zurich : Gisberger, 1939. Vol.

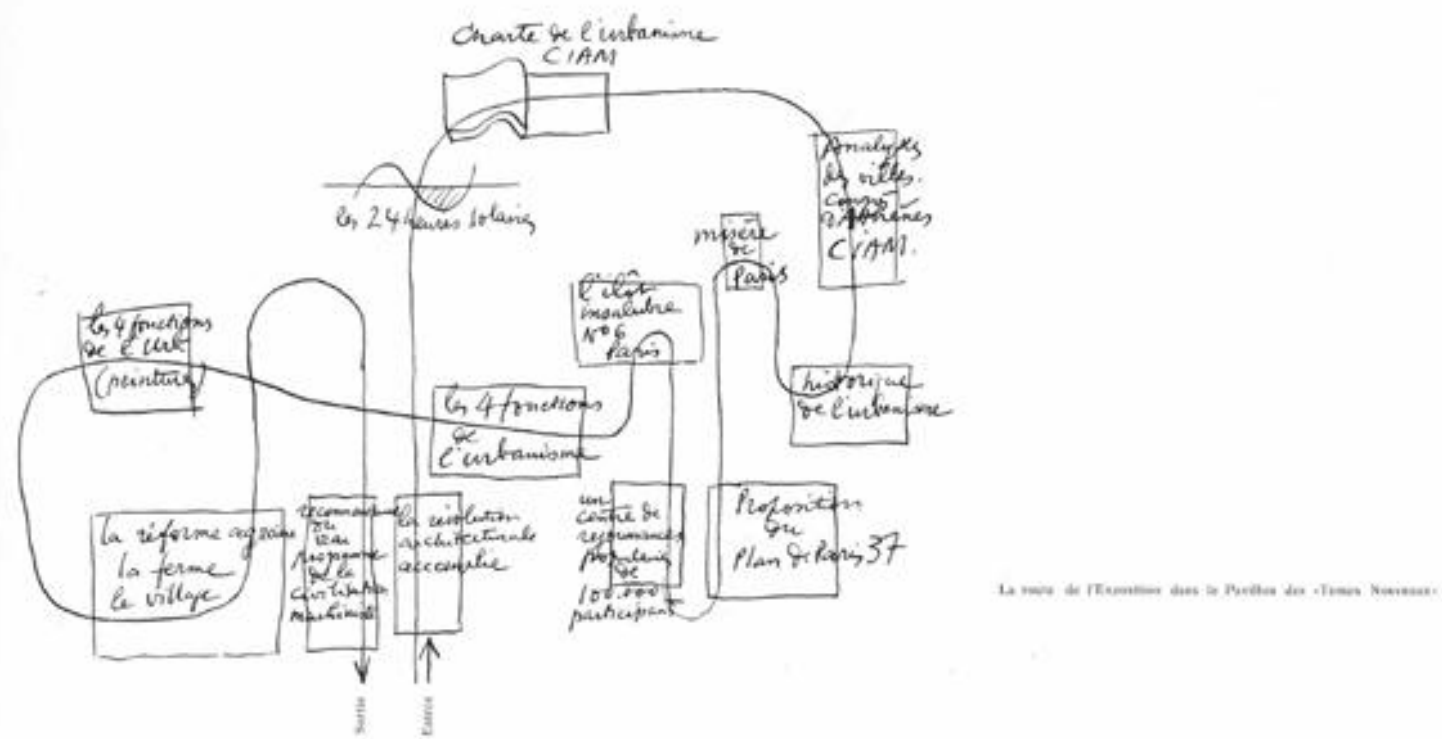

14. Le Corbusier et Pierre Jeanneret, Pavillon des Temps nouveaux, Expo 37, Projet D, la “ route de l'exposition ”. In Boesiger, Willy : Le Corbusier et Pierre Jeanneret, OEuvre complète. 1934-1938. Zurich : Gisberger, 1939. Vol. 3. p. 164. 

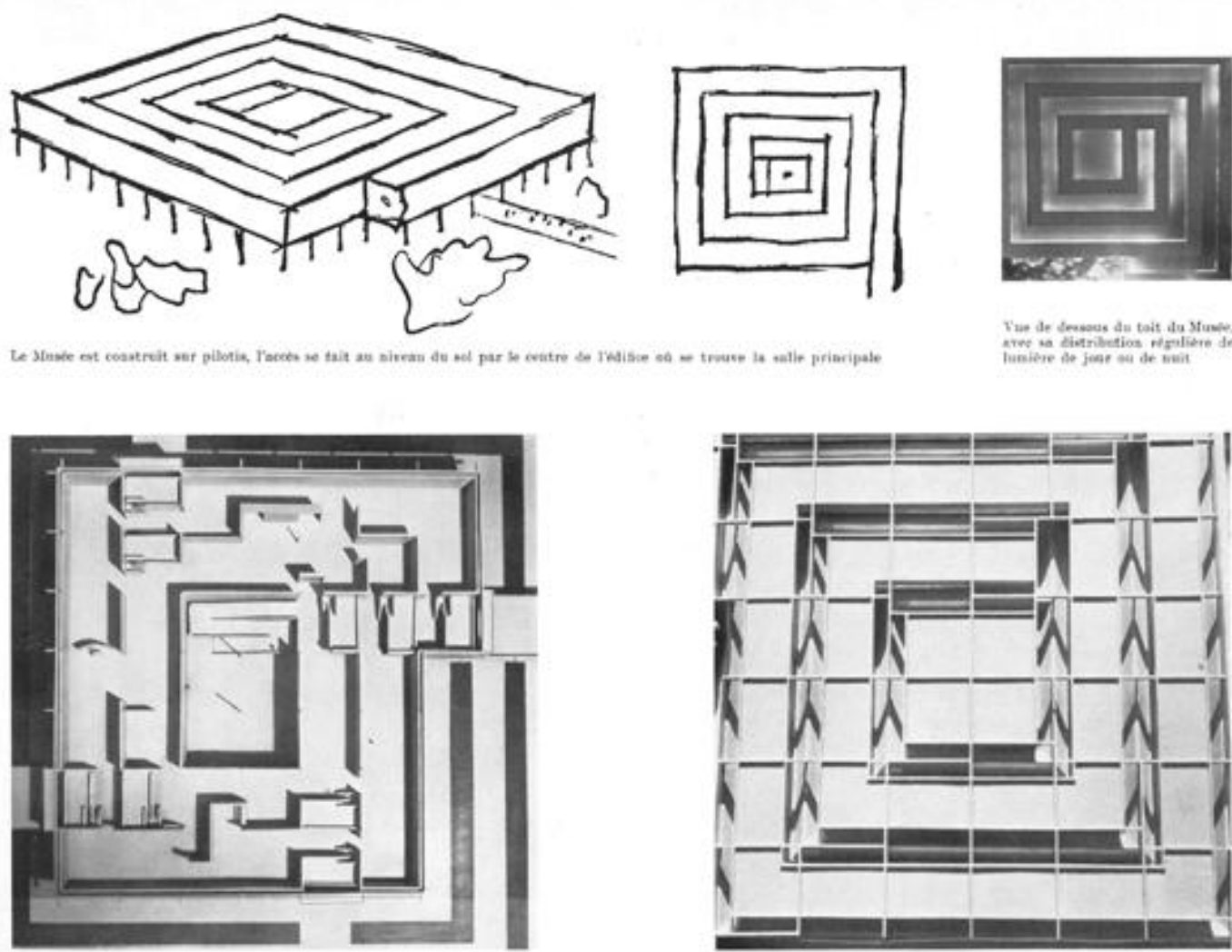

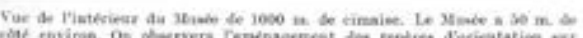

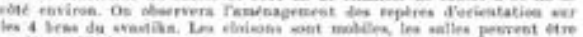

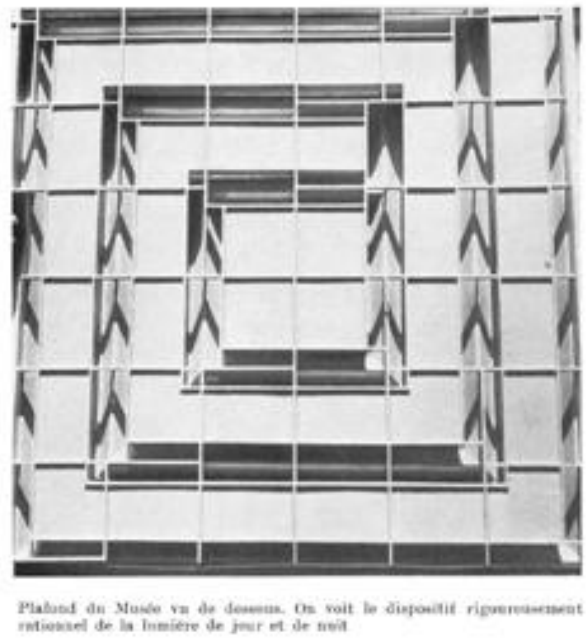

17

15. Le Corbusier et Pierre Jeanneret, Musée à croissance illimitée, 1939. In Boesiger, Willy (dir.) : Le Corbusier et Pierre Jeanneret, OEuvre complète. 1938-1946. Zurich : Gisberger, 1946. Vol. 4. p. 17.

Le projet D, le Pavillon des Temps nouveaux, est une transposition de ces réflexions sous la forme d'une structure éphémère, adaptée à un programme d'éducation populaire et non plus lié à un programme muséal. Les stratégies du parcours à travers les modules constructifs répartis dans l'espace sont semblables.

\section{Le Musée à croissance illimitée, 1939, l'aboutissement d'un modèle}

Le projet du Musée à croissance illimité marque l'incorporation des avancées et des expérimentations effectuées avec les avant-projets pour l'expo 37 dans un bâtiment générique, qui peut être implémenté dans n'importe quel site favorable. Le choix de Philippeville, aujourd'hui Skikda, en Algérie, comme lieu pour le musée dans le volume 4 de l'Euvre complète est une affirmation de la valeur de ready-made du projet, prêt à être construit non seulement en France, mais aussi à être exporté sur d'autres continents. Il porte aussi la marque de la stratégie que Le Corbusier applique pour promouvoir ses projets : Paul Cuttoli, maire radicalsocialiste de la ville et sénateur de Constantine a considérablement contribué au développement de la communauté qu'il gère, faisant construire de nombreuses infrastructures, écoles ou hôpitaux ${ }^{16}$. Marie Cuttoli, sa femme, avait développé une production locale de tapis noués utilisant le savoir-faire traditionnel et initié

\footnotetext{
${ }^{16}$ Sur Paul et Marie Cuttoli voir : http://fse.castanet.free.fr/8_cuttolipaul.html et https://www.facebook.com/media/set/?set=a.477357675671176.1073741848.281519041921708\&type=3.
} 
des collaborations avec des artistes comme Jean Lurçat ou Picasso qui rencontrèrent un véritable succès. Elle collabore régulièrement avec Le Corbusier à partir de 1936 pour l'édition de tapisseries. Les conditions auraient pu être ainsi réunies pour que le projet se concrétise...

Les trois musées construits par l'architecte à partir de 1951 à Tokyo, Ahmedabad et Chandigarh sont des interprétations du modèle adaptées aux contraintes particulières de la commande et des lieux ${ }^{17}$. L'idée de la croissance de la structure dans le temps est mise entre parenthèses.

\section{L'héritage du conflit mondial et la naissance du complexe muséal à la Porte Maillot, 1949- 1951}

Aucun projet concret ne voit le jour entre 1939 et 1945, mais de nombreuses discussions qui dépassent largement le milieu architectural et artistique sont menées sur la question des musées. La politique 'culturelle' de l'occupant allemand, le pillage systématique des collections et leur transfert vers l'Allemagne comme la vente, voire la destruction des œuvres déclarées "dégénérées", qui ont touché aussi bien les collectionneurs privés que les collections des musées nationaux, ont créé une situation d'urgence qui affecte tous les milieux culturels ${ }^{18}$. Avec la perspective de la défaite de l'Allemagne nazie et le retour des œuvres spoliées en France, toute l'organisation et la répartition des œuvres dans les musées français doit être repensée. Jean Cassou a été rétabli dans ses fonctions de directeur du Musée national d'Art moderne à partir de 1945. Il avait été nommé directeur du Musée national d'art moderne en 1940, mais n'occupa ce poste que quelques mois avant d'être nommé responsable du dépôt national du château de Compiègne. Son ouvrage sur $L e$ Pillage par les Allemands des auvres d'art [...] en France ${ }^{19}$ et son engagement en tant que conservateur en font une des chevilles ouvrières de la réorganisation des musées français. Il est proche de Le Corbusier et va lui apporter un soutien indéfectible. Il a préfacé Urbanisme en 1925 et fait partie comme lui des auteurs du recueil La Querelle du réalisme, paru en 1936, recueil qui propose un état des lieux sur le rôle de l'artiste et des œuvres d'art dans la société. Il signera également l'avant-propos du catalogue de l'exposition Le Corbusier : Euvres plastiques, du 17 novembre 1953 au 31 janvier 1954 au Musée national d'art moderne, à Paris. En 1964, c'est encore lui qu'André Malraux charge de superviser la rédaction du programme du Musée du $\mathrm{XX}^{\mathrm{e}}$ siècle qui servira de base au projet de l'architecte. Son nom apparaît de manière récurrente dans les organigrammes que Le Corbusier prépare pour son projet pour un Centre expérimental des arts majeurs pour la Porte Maillot en $1949-1950^{20}$.

\footnotetext{
${ }^{17}$ Sur la réalisation des trois musées voir : Mory, Pascal : "Le Corbusier and the Syndrome of the Museum ". Op. cit. [note 1].

${ }^{18}$ Sur la question de la politique culturelle du $\mathrm{III}^{\mathrm{e}}$ Reich, voir l'ouvrage de référence de Bertrand Dorléac, Laurence : L'art de la défaite, 1940-1944. Paris : Seuil, 1993.

${ }^{19}$ Cassou, Jean : Le pillage par les Allemands des xuvres d'art et des bibliothèques appartenant à des Juifs en France. Paris : Éd. du Centre, 1947. L'ouvrage est cité par Schulmann, Didier: "Spoliation des œuvres d'art, France (1940-1944)". In Encyclopedia universalis, http://www.universalis.fr/encyclopedie/spoliation-des-oeuvres-d-art-france/.

${ }^{20}$ Le Corbusier : "Synthèse des Arts majeurs. Projet pour une exposition à installer à la Porte Maillot 1950 ”. In Boesiger, Willy (dir.) : Le Corbusier. Euvre complète. 1946-1952. Zurich: Gisberger, 1953. Vol. 5. pp. 67-71 et Dumont d'Ayot, Catherine ; Benton, Tim : Le Pavillon de Le Corbusier pour Zurich. Zurich : Lars Müller Publishers - IDB, ETH Zürich, 2013.
} 


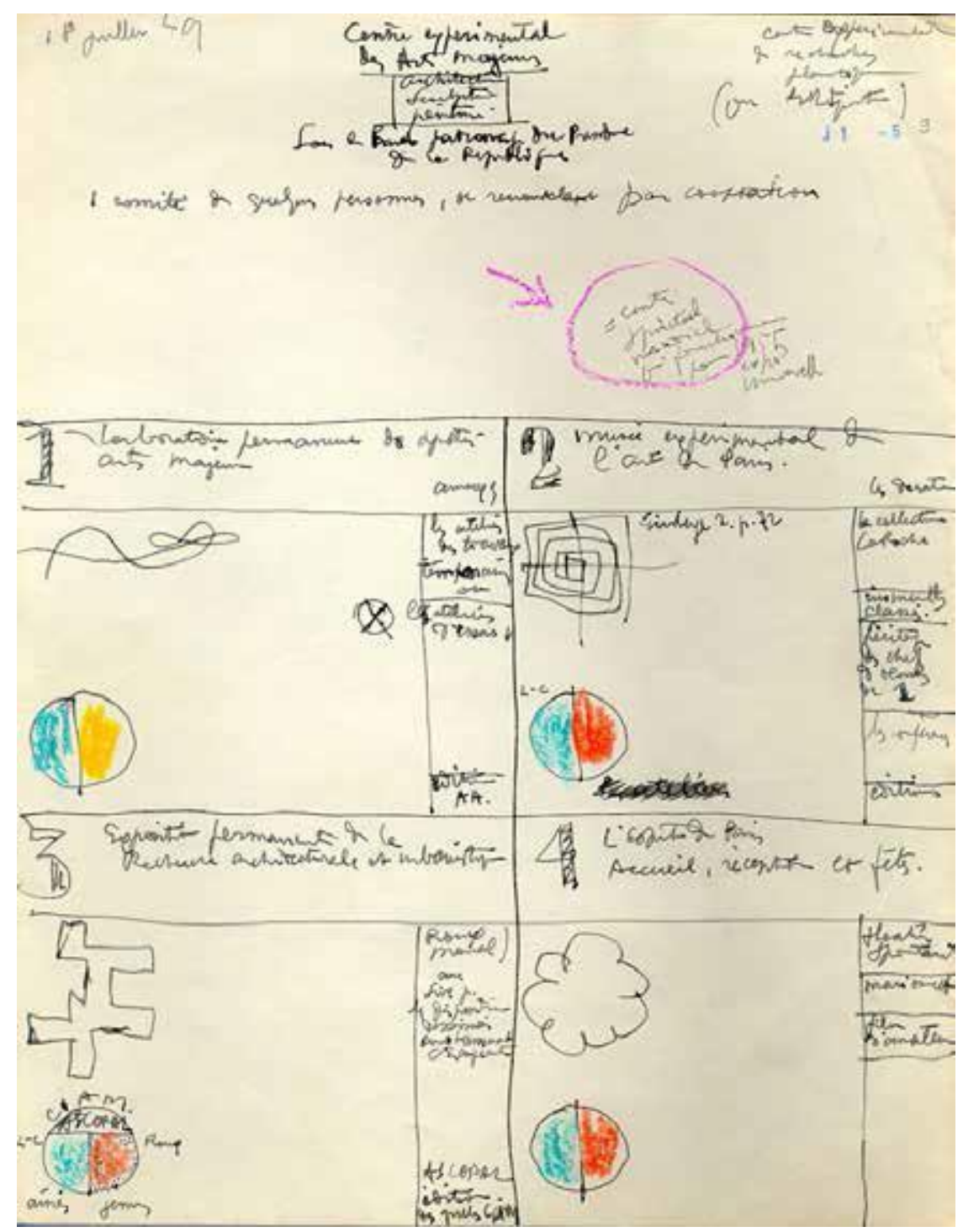

16. Le Corbusier, Centre expérimental des arts majeurs, Porte Maillot, 1949-1950, schéma d'organisation des quatre différentes structures proposées, FLC J1-5-9-001.

Ce projet pour la Porte Maillot est une réponse à l'élargissement de la compréhension du rôle du musée et du statut des œuvres exposées. Ce n'est plus un bâtiment unique qui répond au programme du musée, mais un véritable complexe muséal constitué de parties différenciées : le Laboratoire permanent des arts majeurs, le Musée expérimental de l'art de Paris, une exposition permanente architecturale et urbanistique et l' "Esprit de Paris. Accueil réception et fêtes ". Cette stratégie qui consiste à proposer une sorte de parc d'attraction culturel permet d'élargir le champ d'activité et le public visé, plaçant les ambitions pédagogiques d'une "éducation populaire " au cœur du projet. Le deuxième point du programme, le Musée expérimental de l'art de Paris vient en réponse au manque de représentation des avant-gardes, notamment françaises, dans les collections publiques. Il s'agit d'une nouvelle occurrence du musée à croissance illimitée, cette fois destiné à un musée dont la collection aurait dû abriter la donation Raoul La Roche.

Sans réels appuis politiques ni financiers, le programme se réduit rapidement au seul "Laboratoire permanent de synthèse des Arts majeurs ", structure légère destinée aux expositions temporaires, qui prend dès 1950 la forme iconique d'une toiture monumentale constituée par un double parapluie-parasol inversé. Le passage du premier projet au second a incité Le Corbusier à abandonner l'idée d'un pavillon temporaire pour 
élaborer un projet "plus construit ", un modèle reproductible dans différents lieux. C'est une forme de readymade qui permet à la fois la reproduction du modèle architectural et l'itinérance des expositions.

\section{Les pavillons d'expositions temporaires pour Zurich et Stockholm}

Le Corbusier a l'opportunité de développer en détail le concept du pavillon d'exposition grâce aux commandes de la galeriste zurichoise Heidi Weber et du collectionneur suédois Theodor Ahrenberg entre 1960 et 1965. Le long parcours en spirale des grands musées est remplacé par une double boucle distendu sur deux niveaux dont la géométrie renvoie au symbole mathématique de l'infini $\infty$. Cette stratégie lui permet de déjouer la petite dimension des structures en offrant un parcours continu rythmé par des changements de direction et d'ouverture sur le paysage. Pour le projet du Zurichhorn, les esquisses des avant-projets montrent le long travail de définition du cheminement intérieur. Les positions respectives de la rampe et de l'escalier font l'objet de nombreuses variantes : la rampe, initialement intégrée à l'intérieur du volume, dans le sens de la longueur, est redéployée à l'extérieur, perpendiculairement au volume principal, ce qui permet un allongement significatif du parcours et un accès beaucoup plus spectaculaire à la terrasse, dans l'axe du panorama du lac. Le projet du Palais Ahrenberg impose une élaboration spécifique : il est prévu pour abriter trois ensembles d'œuvres de trois grands artistes : Picasso, Matisse et Le Corbusier, de même qu'un espace pour des expositions temporaires. Le Corbusier veut trois salles d'importance semblable, chacune avec une double hauteur. La manière de diviser l'espace intérieur du pavillon très fortement déterminé par la géométrie de la toiture double en trois salles est un réel défi qui donne lieu à de nombreuses études - Guillermo Jullian de la Fuente, en particulier, y consacre de nombreuses esquisses. La stratégie de mise en scène du parcours du spectateur à l'intérieur de la structure trouve son aboutissement dans le pavillon de Zurich, cette fois libérée de la contrainte d'une collection permanente et ouverte à des usages multiples. Les modules du 226x226x226 semblent avoir permis à Le Corbusier de réaliser son souhait de la fusion des nécessités du logis et de celles de l'exposition en structure parfaitement flexible et adaptable.

\section{La pratique de l'exposition}

Au début de sa carrière, Le Corbusier s'est intéressé à la manière d'exposer des œuvres dans le cadre des projets d'ateliers d'artistes ou des maisons de collectionneurs. Il s'agit d'un rapport intime et privilégié à l'œuvre d'art, qui n'engage que l'artiste ou un connaisseur averti. Ce rapport est exclusif, pour la villa de Raoul La Roche Le Corbusier propose un meuble central pour ranger les tableaux de la collection et choisir la toile exposée dans un face à face exclusif, modèle qui se révélera insatisfais ant pour le collectionneur. Les accrochages se font toujours dans la petite échelle domestique. L'autre modèle, celui vécu et pratiqué par le créateur lui-même, est celui de l'atelier d'artiste. C'est le programme de l'un de ses premiers projets parisiens en 1922 pour Amédée Ozenfant. Mais c'est surtout le rapport à l'œuvre tel qu'il l'expérimente quotidiennement dans son propre travail de peintre qui influence sa compréhension des nécessités de l'exposition. L'atelier est le lieu de la confrontation productive à un choix d'œuvres déterminé en fonction de l'actualité du travail. Cette forme de l'exposition est au cœur du travail, elle est soumise à des remaniements incessants et ne possède pas de forme fixe, c'est un processus en mouvement. Elle est assujettie à la volonté de l'artiste de s'entourer à chaque moment des œuvres susceptibles d'accompagner son travail, propice au dialogue avec ses propres œuvres, mais aussi des œuvres " amies " qu'il possède, celles de Fernand Léger ou Jacques Lipschitz par exemple, sans oublier les “objets à réaction poétique ", ramassés au gré du hasard et des voyages. 


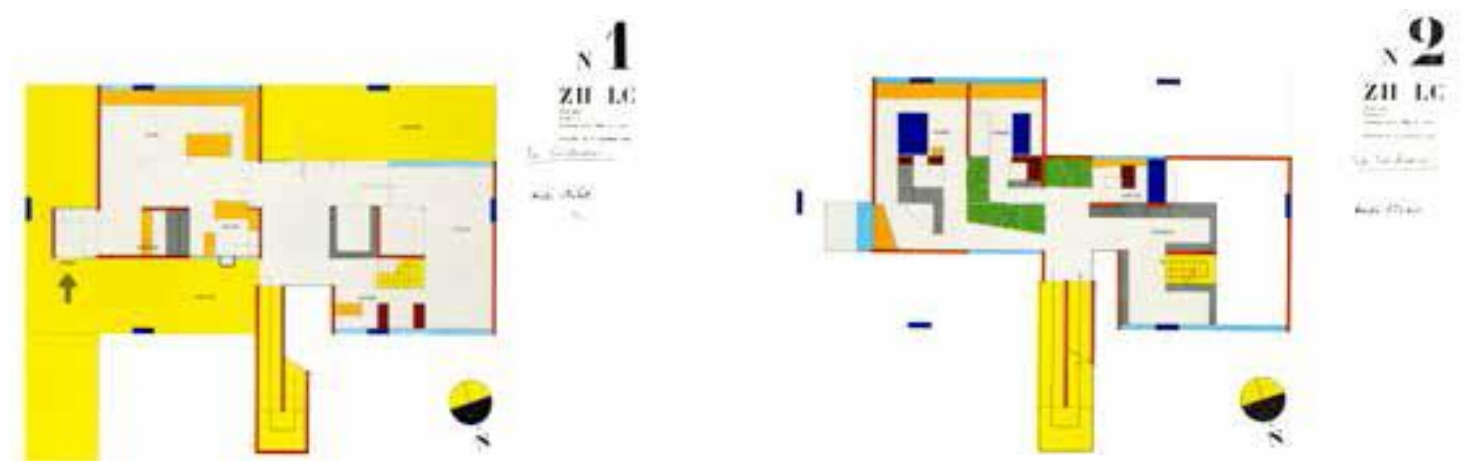

17. Le Corbusier, Pavillon d'exposition pour Heidi Weber, Zurich, décembre 1961, premier permis de construire, premier et second niveaux, à droite, l'atelier, à gauche, le logis. Stadt Zürich, Hochbauamtdepartement, Planauflage / Archiv.

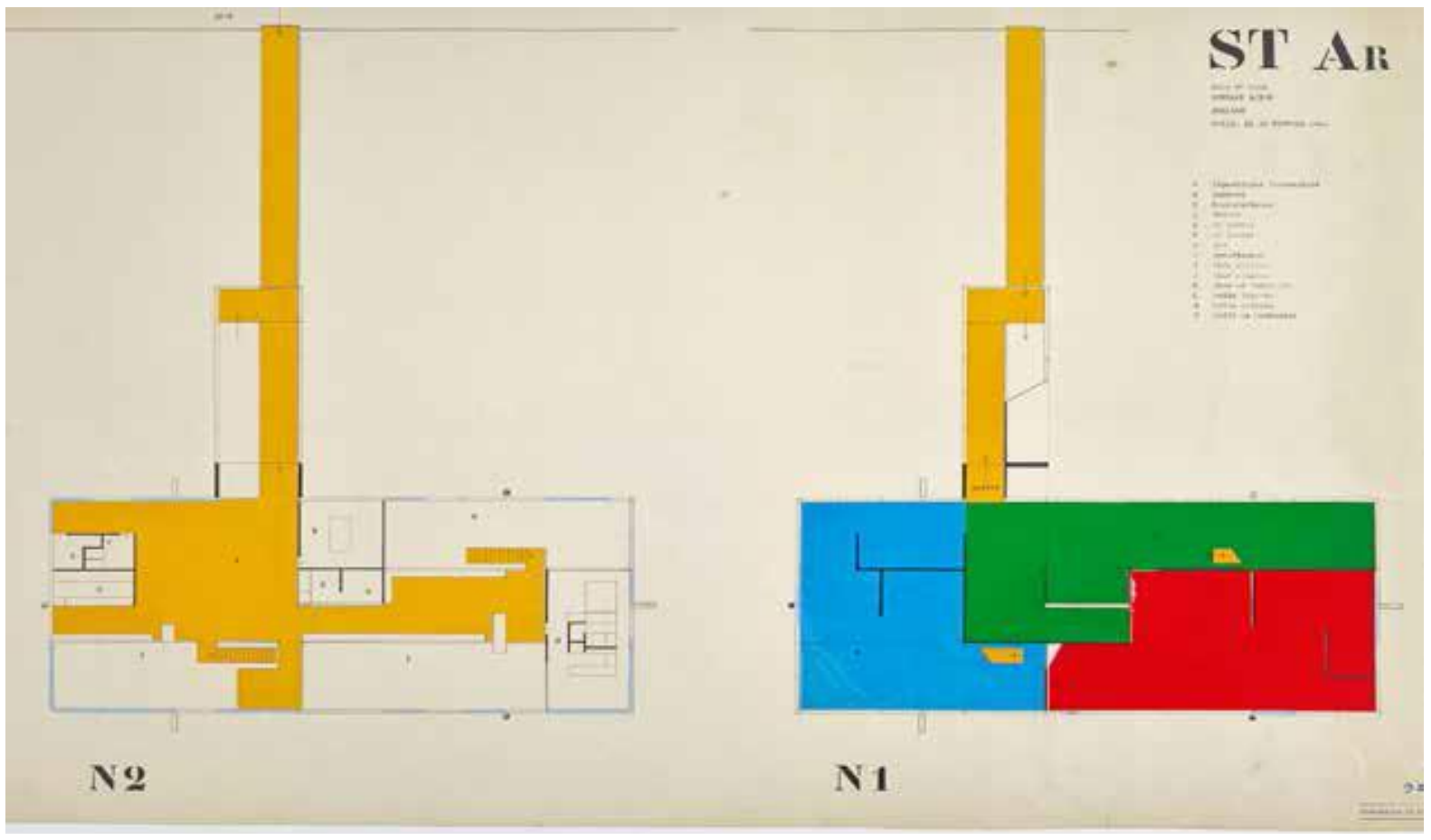

18. Le Corbusier, Palais Ahrenberg, Stockholm, janvier 1962, avant-projet, second et premier niveaux, en jaune, accès et exposition temporaire, en bleu $(\mathrm{J}+\mathrm{M})$ Matisse, en rouge $(\mathrm{I}+\mathrm{L})$ Picasso, en vert $(\mathrm{K}+\mathrm{N})$ Le Corbusier, FLC 25047B.

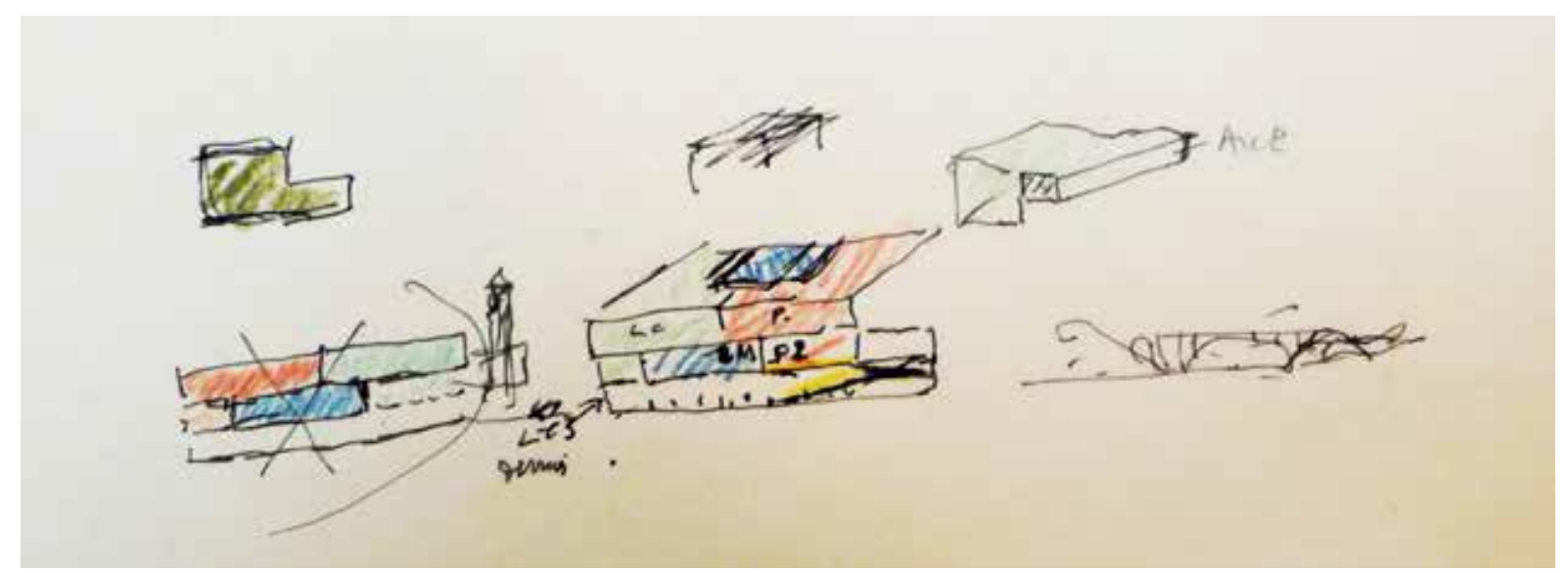

19. Le Corbusier, Palais Ahrenberg, Stockholm, janvier 1962, étude de la disposition des trois salles Matisse, Picasso et Le Corbusier, Centre canadien d'architecture, fonds Guillermo Jullian de la Fuente, DR 1993:0136:0004. 
Très tôt aussi, Le Corbusier expose dans les galeries, en tandem avec Amédée Ozenfant pendant la période puriste, puis seul. Dès 1937, avec l'exposition au Kunsthaus de Zurich ${ }^{21}$, il fait l'expérience de devoir montrer ses propres œuvres dans un musée. Par ses dimensions et par son public beaucoup plus diversifié, le musée rompt avec cette approche intimiste et oblige à concevoir une approche plus pédagogique et une organisation spatiale à même de gérer le rapport simultané à de nombreuses œuvres. En 1940, l'exposition de "La France d'outremer " qui s'est tenue au Grand Palais lui permet de se confronter à la très grande échelle du lieu, mais c'est surtout avec l'exposition de son propre travail au Musée national d'art moderne de la ville de Paris qu'il se heurte à la question de l'échelle des salles d'exposition, expérience qu'il relate dans le Modulor 2 sous le titre "Transformation d'une salle inhumaine 22 ". Il introduit des éléments de partitions de l'espace au format 226×226×226 afin de "rétablir par une initiative efficace un contact entre spectateurs et æuvres (tableaux, sculptures, documents graphiques) ${ }^{23}$ ". C'est ce module qui remplace la trame initiale de $7 \times 7 \mathrm{~m}$ des musées et qui est mis en œuvre dans les scénographies et les aménagements intérieurs de ses expositions successives, puis, finalement, dans le Pavillon de Zurich grâce au système constructif en cornières soudées, matérialisation dans l'espace de cette trame abstraite idéale.

\section{Conclusion}

Le projet du Musée d'Art contemporain de 1931 constitue un changement de paradigme qui se répercute dans l'ensemble de l'œuvre de Le Corbusier. Le statut provisoire de la façade prend à contre-pied non seulement l'architecture académique mais aussi les formes compositives issues de la Modernité. L'architecture moderne supprime tout décor de la façade, Le Corbusier franchit le pas suivant et propose une architecture libérée de la charge du dessin même d'une façade. Le projet est littéralement la transcription d'un concept, d'un parcours construit comme une suite de face-à-face avec les œuvres exposées. L'entrée est disposée au centre de la spirale, la façade n'assume plus l'identité visuelle du musée. Cette logique trouvera un aboutissement dans le Carpenter Art Center à Boston, ou le dedans devient le dehors.

La réduction de l'architecture à une trame, une grille tridimensionnelle dans laquelle le programme viendra s'inscrire relève de la même logique : la recherche d'une abstraction maximale. Les dessins et les esquisses restituent cette intention : de nombreux plans se résument à des grilles de points et de flèches, des schéma d'organisation, tandis que certaines perspectives ne montrent pas directement des espaces, mais se concentrent des mises en situation, des confrontations entre le spectateur et l'œuvre. C'est ce rapport spectateur-œuvre qui détermine le projet, non plus une forme symbolique chargée de la représentation d'une institution. Il reste le face à face avec l'œuvre, le parcours et le discours sur le corpus exposé.

\footnotetext{
${ }^{21}$ Voir Navarro Segura, Maria Isabel (dir.), Le Corbusier expose, catalogue d'exposition. Besançon : Musée des Beaux arts et d'archéologie de Besançon - Milan : Silvana Editoriale, 2011.

${ }^{22}$ Le Corbusier, Modulor 2. Boulogne : éditions de l'Architecture d'aujourd'hui, 1955. pp. 275-279.

${ }^{23}$ Ibid.
} 

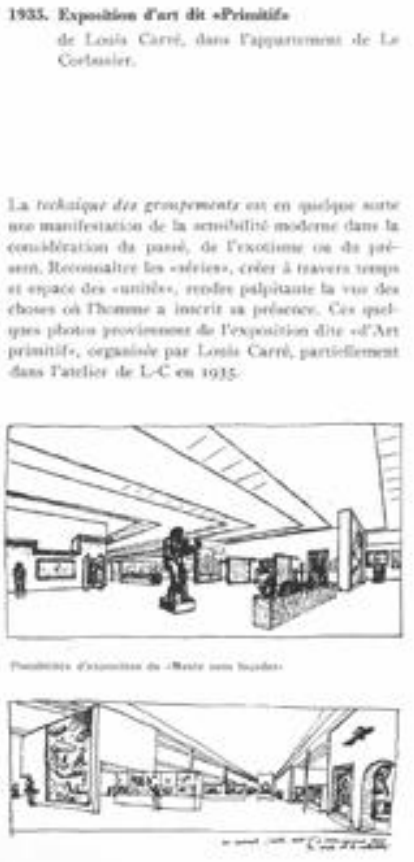
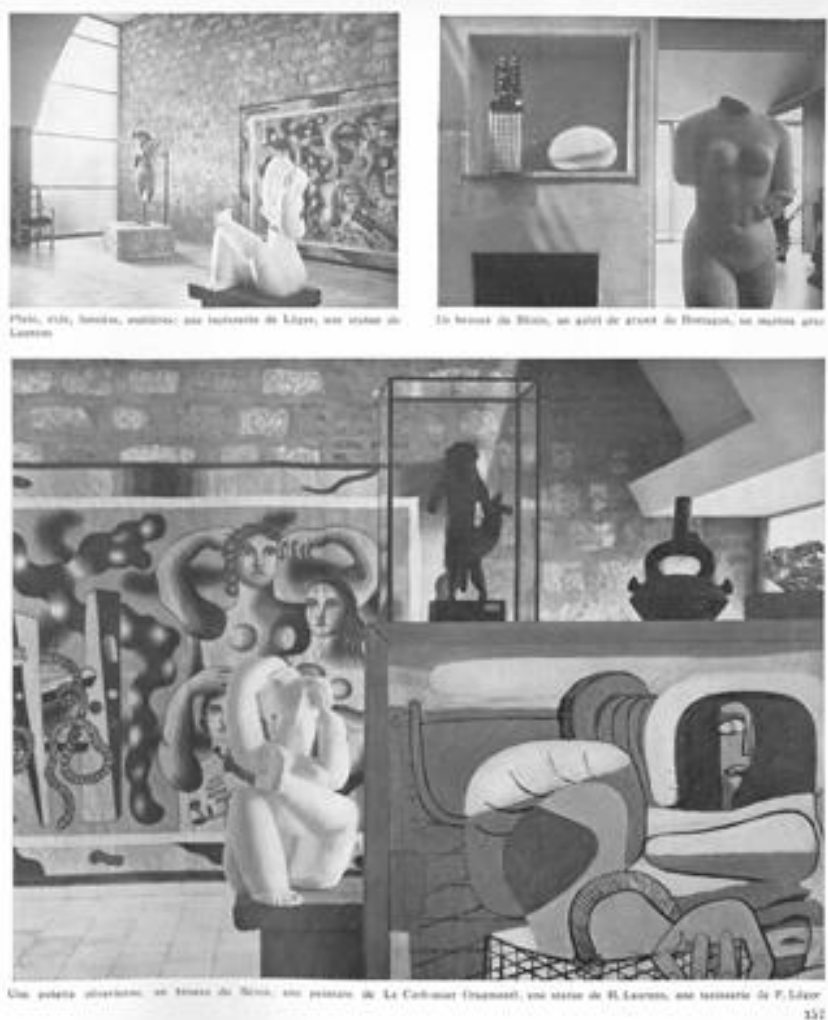

20. Le Corbusier, Exposition des arts dit “primitifs" de Louis Carré dans l'appartement de Le Corbusier. In Boesiger, Willy (dir.) : Le Corbusier et Pierre Jeanneret, OEuvre complète. 1934-1938. Zurich : Gisberger, 1939. Vol. 3. p. 157.
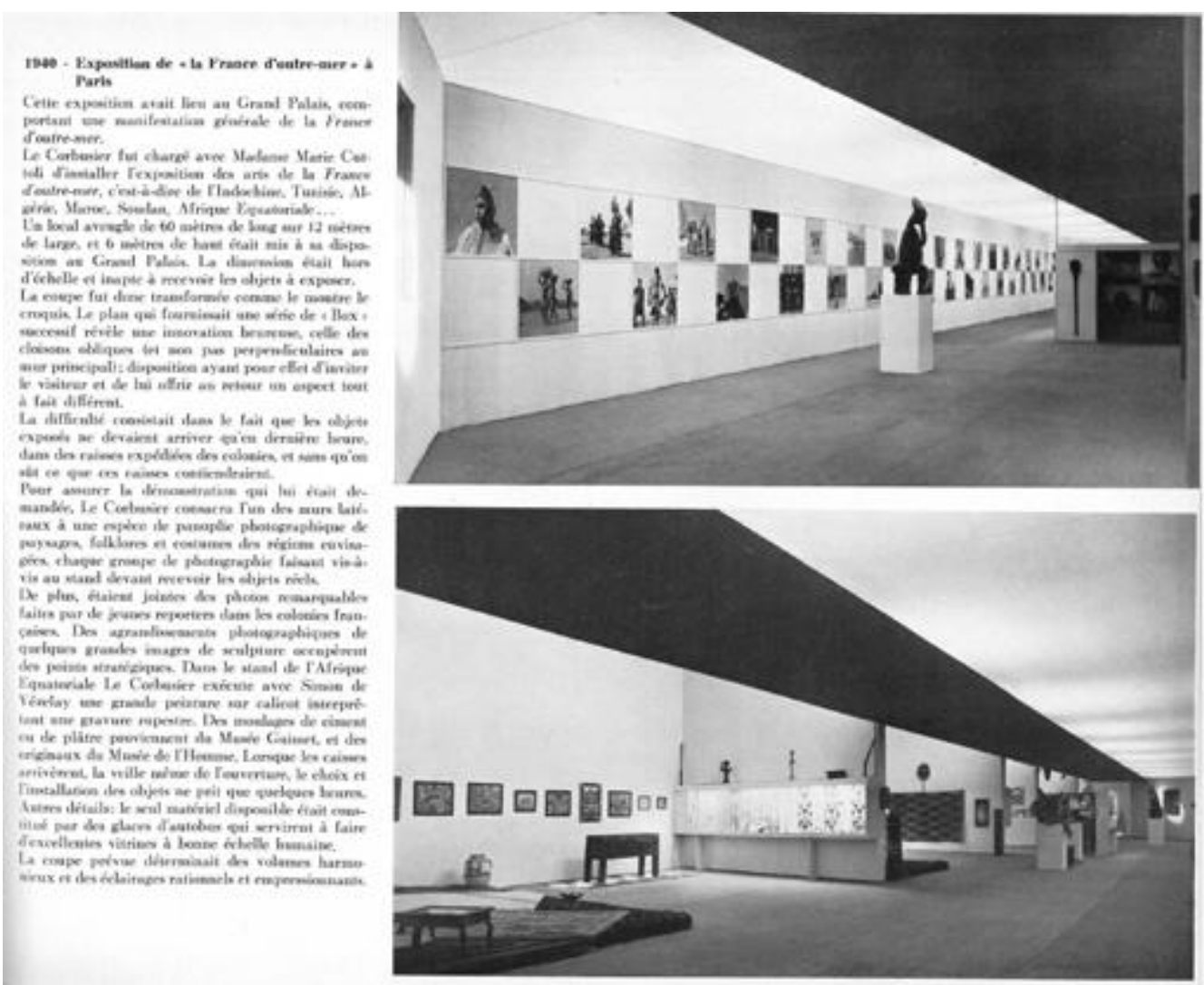

21. Le Corbusier, Exposition de “ la France d'Outre-mer” à Paris. In Boesiger, Willy (dir.) : Le Corbusier et Pierre Jeanneret, OEuvre complète. 1938-1946. Zurich : Gisberger, 1946. Vol. 4. p. 91. 


\section{CAPTIONS / Légendes des illustrations}

1. Le Corbusier et Pierre Jeanneret, Mundaneum, perspective de Le Corbusier montrant le panorama vers le lac Léman et le Mont-Blanc, FLC 32114.

2. Le Corbusier et Pierre Jeanneret, Mundaneum, le Musée mondial, schéma montrant le fonctionnement de la circulation en spirale descendante et des trois nefs destinées aux objets (a), lieux (b) et temps (c) et le chemin de fer qui dessert le magasin continu (d), In Boesiger, Willy; Storonov, Oscar (dir.) : Le Corbusier et Pierre Jeanneret, Euvre complète.1910-1929. Zurich : Gisberger, 1937. Vol. 1, p. 193.

3. Le Corbusier et Pierre Jeanneret, Musée d'art contemporain, Paris, 1930, perspective aérienne. In Boesiger, Willy (dir.) : Le Corbusier et Pierre Jeanneret, Euvre complète. 1929-1934. Zurich : Gisberger, 1935. Vol. 2. p. 72 .

4. Le Corbusier et Pierre Jeanneret, Musée d'art contemporain, Paris, 1930, principe de croissance. In Boesiger, Willy (dir.) : Le Corbusier et Pierre Jeanneret, Euvre complète. 1929-1934. Zurich : Gisberger, 1935. Vol. 2. p. 73.

6. Le Corbusier et Pierre Jeanneret, Musée de la ville et de l'état, Paris, 1934, perspective, FLC 28814.

7. Le Corbusier et Pierre Jeanneret, Expo 37, Projet C, plan et élévation. In Boesiger, Willy (dir.) : Le Corbusier et Pierre Jeanneret, Euvre complète. 1934-1938. Zurich : Gisberger, 1938. Vol. 3. p. 155.

8. Le Corbusier et Pierre Jeanneret, Expo 37, Projet C, perspective à vol d'oiseau, FLC 626.

9. Le Corbusier et Pierre Jeanneret, Expo 37, Projet C, schéma de circulation, FLC 713.

10. Le Corbusier et Pierre Jeanneret, Expo 37, Projet C, schéma d'organisation, FLC 705.

11. Le Corbusier et Pierre Jeanneret, Expo 37, Projet C, schéma d'organisation, FLC 675.

12. Le Corbusier et Pierre Jeanneret, Expo 37, Projet C, perspective, FLC 768

13. Le Corbusier et Pierre Jeanneret, Pavillon des Temps nouveaux, Expo 37, Projet D, élévation, plan et coupe. In Boesiger, Willy (dir.) : Le Corbusier et Pierre Jeanneret, Euvre complète. 1934-1938. Zurich : Gisberger, 1939. Vol. 3. p. 160.

14. Le Corbusier et Pierre Jeanneret, Pavillon des Temps nouveaux, Expo 37, Projet D, la "route de l'exposition ”. In Boesiger, Willy : Le Corbusier et Pierre Jeanneret, Euvre complète. 1934-1938. Zurich : Gisberger, 1939. Vol. 3. p. 164.

15. Le Corbusier et Pierre Jeanneret, Musée à croissance illimitée, 1939. In Boesiger, Willy (dir.) : Le Corbusier et Pierre Jeanneret, Euvre complète. 1938-1946. Zurich : Gisberger, 1946. Vol. 4. p. 17.

16. Le Corbusier, Centre expérimental des arts majeurs, Porte Maillot, 1949-1950, schéma d'organisation des quatre différentes structures proposées, FLC J1-5-9-001.

17. Le Corbusier, Pavillon d'exposition pour Heidi Weber, Zurich, décembre 1961, premier permis de construire, premier et second niveaux, à droite, l'atelier, à gauche, le logis. Stadt Zürich, Hochbauamtdepartement, Planauflage / Archiv.

18. Le Corbusier, Palais Ahrenberg, Stockholm, janvier 1962, avant-projet, second et premier niveaux, en jaune, accès et exposition temporaire, en bleu $(\mathrm{J}+\mathrm{M})$ Matisse, en rouge $(\mathrm{I}+\mathrm{L})$ Picasso, en vert $(\mathrm{K}+\mathrm{N}) \mathrm{Le}$ Corbusier, FLC 25047B.

19. Le Corbusier, Palais Ahrenberg, Stockholm, janvier 1962, étude de la disposition des trois salles Matisse, Picasso et Le Corbusier, Centre canadien d'architecture, fonds Guillermo Jullian de la Fuente, DR 1993:0136:0004.

20. Le Corbusier, Exposition des arts dit “primitifs” de Louis Carré dans l'appartement de Le Corbusier. In Boesiger, Willy (dir.) : Le Corbusier et Pierre Jeanneret, Euvre complète. 1934-1938. Zurich : Gisberger, 1939. Vol. 3. p. 157.

21. Le Corbusier, Exposition de “la France d'Outre-mer” à Paris. In Boesiger, Willy (dir.) : Le Corbusier et Pierre Jeanneret, Euvre complète. 1938-1946. Zurich : Gisberger, 1946. Vol. 4. p. 91. 


\section{Bibliographie}

Bertrand Dorléac, Laurence : L'art de la défaite, 1940-1944. Paris : Seuil, 1993.

Boesiger, Willy (dir.) : Le Corbusier et Pierre Jeanneret, Euvre complète. 1938-1946. Zurich : Gisberger, 1946. Vol. 4. pp. 91-92.

Cohen, Jean-Louis : Le Corbusier et la mystique de l'URSS. Bruxelles : Mardaga, 1987.

Courtiau, Catherine : "La Cité internationale". In Charollais, Isabelle ; Ducret, André (dir.) : Le Corbusier à Genève 1922-1932. Projets et réalisations, catalogue d'exposition. Lausanne : Payot, 1987. pp. 53-69.

Dumont d'Ayot, Catherine ; Benton, Tim : Le Pavillon de Le Corbusier pour Zurich. Zurich : Lars Müller Publishers - IDB, ETH Zürich, 2013.

Gresleri, Giuliano : "Le Mundaneum. Lecture d'un projet". In Charollais, Isabelle ; Ducret, André (dir.) : Le Corbusier à Genève 1922-1932. Projets et réalisations, catalogue d'exposition. Lausanne : Payot, 1987. pp. 7078.

Gresleri, Giuliano : "Mundaneum”. In Lucan, Jacques (dir.) : Le Corbusier, une encyclopédie. Paris : Éditions du Centre Pompidou, 1987. pp. 261-293.

Le Corbusier : “ Défense de l'architecture ”. In L'Architecture d'aujourd'hui. 1933. º 10. pp. 38-61.

Le Corbusier: "Mundaneum, 1929 ". In Boesiger, Willy; Storonov, Oscar (dir.) : Le Corbusier et Pierre Jeanneret. Euvre complète. 1910-1929. Zurich : Gisberger., 1937. 2édition. Vol. 1. p. 194.

Le Corbusier: "Musée d'art contemporain”, Paris, 1932. In Boesiger, Willy (dir.) : Le Corbusier et Pierre Jeanneret, Euvre complète. 1929-1934. Zurich : Gisberger, 1935. Vol. 2. pp. 72-73.

Le Corbusier : “Plans pour les musées de la Ville et de l'Etat à Paris ”, Paris, 1932. In Bill, Max (dir.) : Le Corbusier et Pierre Jeanneret, Euvre complète. 1934-1938. Zurich : Gisberger, 1953. Vol. 3. pp. 82-89.

Le Corbusier ; Jeanneret, Pierre : “Exposition de "la France d'Outre-mer " à Paris ". In Boesiger, Willy (dir.) : Le Corbusier et Pierre Jeanneret, CEuvre complète. 1938-1946. Zurich : Gisberger, 1946. Vol. 4. pp. 9192.

Le Corbusier ; Jeanneret, Pierre : "Exposition des arts dit primitifs". In Boesiger, Willy (dir.) : Le Corbusier et Pierre Jeanneret, Euvre complète. 1934-1938. Zurich : Gisberger, 1939. Vol. 3. pp. 156-157.

Le Corbusier; Jeanneret, Pierre: “Musée à croissance illimitée, 1939 ”. In Boesiger, Willy (dir.) : Le Corbusier et Pierre Jeanneret, Euvre complète. 1938-1946. Zurich : Gisberger, 1946. Vol. 4. pp. 17.

Le Corbusier ; Jeanneret, Pierre : "Pour la création à Paris d'un musée des artistes vivants ", In Cahiers d'art. 1931. $\mathrm{N}^{\circ} 1$, p. 9.

Mory, Pascal. "Le Corbusier and the Syndrome of the Museum". In Cohen, Jean-Louis ; Ahrenberg, Staffan (dir.) : Le Corbusier's Secret Laboratory. From Painting to Architecture. Ostfildern : Hatje Cantz, 2013.

Perret, Auguste : “Le musée moderne ”. In Mouseion. Décembre 1929. N 9. pp. 225-235.

Reichlin, Bruno: "Solution élégante". In Lucan, Jacques (dir.) : Le Corbusier, une encyclopédie. Paris : Éditions du Centre Pompidou, 1987. pp. 369-377.

Teige, Karel : “Mundaneum ”. In Stavba. Avril 1929. ํㅜ 10. pp. 145-155. La traduction anglaise est parue dans Opposition. Octobre 1974. № 4. pp. 80-90. 